Research Paper

\title{
The P2RX7B splice variant modulates osteosarcoma cell behaviour and metastatic properties
}

\author{
Luke Tattersall $^{\mathrm{a}}$, Karan M. Shah ${ }^{\mathrm{a}}$, Darren L. Lath ${ }^{\mathrm{a}}$, Archana Singh ${ }^{\mathrm{b}}$, Jennifer M. Down ${ }^{\mathrm{a}}$, Elena De Marchi ${ }^{\mathrm{c}}$, \\ Alex Williamson ${ }^{a}$, Francesco Di Virgilio ${ }^{c}$, Dominique Heymann ${ }^{\mathrm{d}}$, Elena Adinolfi ${ }^{\mathrm{c}}$, William D. Fraser ${ }^{\mathrm{e}, \mathrm{f}}$, \\ Darrell Green ${ }^{\mathrm{e}}$, Michelle A. Lawson ${ }^{\mathrm{a}}$, Alison Gartland ${ }^{\mathrm{a}, *}$
}

\footnotetext{
${ }^{a}$ The Mellanby Centre for Musculoskeletal Research, Department of Oncology and Metabolism, The University of Sheffield, UK

${ }^{\mathrm{b}}$ School of Biological Sciences, University of East Anglia, Norwich, UK

${ }^{\mathrm{c}}$ Department of Medical Sciences, Section of Experimental Medicine, University of Ferrara, Italy

d Université de Nantes, Institut de Cancérologie de l'Ouest, Saint-Herblain, France

e Norwich Medical School, University of East Anglia, Norwich Research Park, Norwich, UK

${ }^{\mathrm{f}}$ Clinical Biochemistry, Norfolk and Norwich University Hospital, Norwich Research Park, Norwich, UK
}

\section{A R T I C L E I N F O}

\section{Article history:}

Received 10 August 2021

Revised 18 October 2021

Accepted 19 October 2021

Available online 20 October 2021

\section{Keywords:}

ATP

Bone cancer

Osteosarcoma

P2RX7B

Purinergic signalling

\begin{abstract}
A B S T R A C T
Background: Osteosarcoma (OS) is the most common type of primary bone cancer affecting children and adolescents. OS has a high propensity to spread meaning the disease is often incurable and fatal. There have been no improvements in survival rates for decades. This highlights an urgent need for the development of novel therapeutic strategies. Here, we report in vitro and in vivo data that demonstrates the role of purinergic signalling, specifically, the B isoform of the purinergic receptor P2RX7 (P2RX7B), in OS progression and metastasis.

Methods: TE85 and MNNG-HOS OS cells were transfected with P2RX7B. These cell lines were then characterised and assessed for proliferation, cell adhesion, migration and invasion in vitro. We used these cells to perform both paratibial and tail vein injected mouse studies where the primary tumour, bone and lungs were analysed. We used RNA-seq to identify responsive pathways relating to P2RX7B.

Results: Our data shows that P2RX7B expression confers a survival advantage in TE85 + P2RX7B and MNNG-HOS + P2RX7B human OS cell lines in vitro that is minimised following treatment with A740003, a specific P2RX7 antagonist. P2RX7B expression reduced cell adhesion and P2RX7B activation promoted invasion and migration in vitro, demonstrating a metastatic phenotype. Using an in vivo OS xenograft model, MNNG-HOS + P2RX7B tumours exhibited cancer-associated ectopic bone formation that was abrogated with A740003 treatment. A pro-metastatic phenotype was further demonstrated in vivo as expression of $\mathrm{P} 2 \mathrm{RX} 7 \mathrm{~B}$ in primary tumour cells increased the propensity of tumour cells to metastasise to the lungs. RNA-seq identified a novel gene axis, FN1/LOX/PDGFB/IGFBP3/BMP4, downregulated in response to $\mathrm{A} 740003$ treatment.

Conclusion: Our data illustrates a role for P2RX7B in OS tumour growth, progression and metastasis. We show that P2RX7B is a future therapeutic target in human OS.

( 2021 Published by Elsevier GmbH. This is an open access article under the CC BY-NC-ND license (http://
\end{abstract} creativecommons.org/licenses/by-nc-nd/4.0/).
Abbreviations: ATP, Adenosine Triphosphate; BzATP, Benzoyl ATP; DE, Differentially Expressed; ECM, Extracellular Matrix; EMT, Epithelial-mesenchymal trans ition; FDR, False Discovery Rate; HBSS, Hanks Balanced Salt Solution; LOX, Lysyl Oxidase; MMPs, Matrix Metalloproteinases; OS, Osteosarcoma; PE, Paired End; qRTPCR, Quantitative Reverse Transcription Polymerase Chain Reaction.

* Corresponding author.

E-mail address: a.gartland@sheffield.ac.uk (A. Gartland).

\section{Background}

OS is the most common type of primary bone cancer affecting children and adolescents, it is a rare and often fatal disease [1]. OS is highly resistant to radiotherapy, therefore treatment involves complex chemotherapy and surgery, often amputation. Survival statistics have remained static with no real advances in treatment options for decades. The 5-year overall survival rate has remained static for decades, at $53 \%$ for patients under 40 years of age [2], however, with metastasis this figure decreases to around 20\% [3]. 
These statistics highlight an urgent need for development of novel therapeutic strategies for OS treatment.

Purinergic receptors are expressed and play a role in the progression of several cancers [4]. Several studies have identified adenosine triphosphate (ATP) to be at a high concentration in the tumour microenvironment $[5,6]$, yet undetectable in surrounding healthy tissue [7]. These findings are particularly pertinent in the case of the bone tumour microenvironment where purinergic receptors are known to be expressed by bone cells that play a key role in normal bone physiology [8,9] and where mechanical loading can stimulate ATP release from bone cells [9-11]. ATP when broken down to adenosine can act as an immunosuppressant and chemotherapy treatments can cause the release of ATP from dying cells [12]. This release can modify the tumour microenvironment in favour of cancer growth and potentially result in disease progression. Purinergic receptor P2RX7 is an ATP gated ion channel and has received an increasing amount of attention due to both in vitro and in vivo evidence of its overexpression and function in carcinogenesis of a range of different tumours, including bone sarcomas [4,12-19]. P2RX7 contributes to various oncogene-like properties such as enhancing tumour cell growth [20] and metabolic activity, increasing invasiveness [21] and metastatic potential by acting in an autocrine/paracrine manner on surrounding cells and by acting as a stimulant for the release of angiogenic factors [14]. Nine different naturally occurring human P2RX7 splice variants (P2RX7A to P2RX7J) have been identified with each showing differing biological characteristics [22,23]. P2RX7 isoform expression, phenotype and level of activation can determine the functional role that P2RX7 has in the tumour microenvironment. The full length P2RX7A variant has the unique ability to form a large transmembrane pore in response to high levels of ATP resulting in cell death [23]. P2RX7B preserves an intron between exons 10 and 11 that causes the insertion of a stop codon that eliminates translation of the last 249 amino acids of the $C$ terminus and the addition of an extra 18 amino acids after residue 346 . These alterations still enable receptor stimulation by ATP/Benzoyl ATP (BzATP) and importantly efficacy of P2RX7 antagonists [23]. Of the splice variants, P2RX7B is unique in that it retains its ability to act as a functional ion channel $[23,24]$ and shares a similar tissue distribution as the full length receptor [25]. Due to the truncated nature of the P2RX7B it naturally lacks the pore function with a "nonfunctional" phenotype found to be essential for tumour cell growth [26] and more recently other features including response to treatment, invasion and promotion of metastasis [27]. In lung adenocarcinoma patient samples, high P2RX7B expression correlated with reduced immune cell infiltration, suggesting it could be a potential biomarker [28]. Whilst in acute myeloid leukaemia, P2RX7B expression was increased in relapsing patients and downregulated in patients in remission, suggesting high levels of P2RX7B may contribute towards chemoresistance. This was proposed to be due to the absence of the full-length P2RX7A receptor pore, as when the full length receptor was present chemotherapy uptake was increased. The study further showed that treatment in vivo with combined chemotherapy and P2RX7 antagonist was an effective strategy acting on both isoforms, reducing tumour growth and isoform expression in the primary tumour [29].

Previous studies by our lab and others have shown a potential role for targeting P2RX7 as a therapeutic option in OS $[15,30]$. When assessing different P2RX7 splice variants in TE85 human OS cells P2RX7B provided a growth advantage in vitro. In a tissure microarray of human OS patient samples stained with antibodies specific to the extracellular or c-terminal region of P2RX7, 27\% stained positive for P2RX7B alone. Further analysis of the samples revealed that $\mathrm{P} 2 \mathrm{RX} 7 \mathrm{~B}$ positive osteosarcomas showed a higher cell density and increased Ki67 positivity indicating a relationship between P2RX7B expression and enhanced cell proliferation and that OS phenotypes can be influenced by P2RX7B [15]. More recently it has been suggested that the full length P2RX7A could promote OS cell growth and metastasis both in vitro and in vivo [30]. In this study we have built upon both of these previous studies and explored the role of the specific P2RX7B splice variant in OS both in vitro and in vivo.

\section{Materials and methods}

\subsection{Cell culture and transfections}

The human OS cell lines HOS-TE85 [31] (referred to herein as TE85) and MNNG-HOS [32] were kindly provided by Professor Jim Gallagher (Liverpool, UK) and Professor Dominique Heymann (INSERM, France), respectively. HEK-293 + P2RX7A cells were kindly provided from the laboratory of Dr Elena Adinolfi (Ferrara, Italy). All cell lines were authenticated using STR profiling during the project period/within the last three years. Cell lines were maintained in DMEM $\odot+$ Glutamax $^{\mathrm{TM}}$ (Life Technologies, UK) medium containing $10 \%$ FBS with $1 \%$ penicillin/streptomycin antibiotics (Life Technologies, UK) (referred to as complete medium). TE85 cells previously stably transfected with P2RX7B [15] were maintained in complete culture medium plus $0.2 \mathrm{mg} / \mathrm{ml}$ hygromycin. MNNG-HOS cells containing a plasma membrane luciferase (PmeLUC) from the lab of Professor Francesco Di Virgilio [33] were further transfected with the mammalian expression vector pcDNA3 containing P2RX7B [15] using Lipofectamine and maintained under $0.8 \mathrm{mg} / \mathrm{ml} \mathrm{G} 418$ selection for PmeLUC and $0.2 \mathrm{mg} / \mathrm{ml}$ hygromycin for P2RX7B. All cells were grown at $37^{\circ} \mathrm{C}$ in a humidified $5 \%$ $\mathrm{CO}_{2}$ incubator and were regularly mycoplasma tested to ensure that all experiments were performed with mycoplasma-free cells.

\subsection{Fluo-4 Direct ${ }^{\mathrm{TM}}$ calcium assay}

Changes in intracellular calcium concentrations were measured using Fluo-4 Direct ${ }^{\mathrm{TM}}$ Calcium Assay (ThermoFisher Scientific, UK) in accordance with the manufacturer's instructions. In brief, 15,000 cells were loaded with Fluo- 4 Direct ${ }^{\mathrm{TM}}$ calcium reagent, left to incubate for $1 \mathrm{~h}$ at $37{ }^{\circ} \mathrm{C}$ before being stimulated with $100 \mu \mathrm{M}$ BzATP (Sigma, UK) (based on Agrawal et al 2020, [34]) and then $0.8 \mu \mathrm{M}$ ionomycin (Sigma, UK). Excitation ratio and emission wavelengths were $490 \mathrm{~nm}$ and $525 \mathrm{~nm}$ respectively.

\subsection{Measurement of plasma membrane permeabilization}

Increases in plasma membrane permeability were measured by monitoring ethidium bromide up-take. Briefly,15,000 cells were incubated at $37^{\circ} \mathrm{C}$ with or without $10 \mu \mathrm{M}$ of the $\mathrm{A} 740003$ (Tocris, UK) for $1 \mathrm{~h}$ in Hank's Balanced Salt Solution (HBSS) (ThermoFisher Scientific, UK). Cells were then stimulated with $300 \mu \mathrm{M}$ BzATP (previously shown to activate pore formation [15,34,35]) which was diluted in ultra-pure ethidium bromide to a final concentration of $100 \mu \mathrm{M}$. Induction of P2RX7 pore formation was then detected at $360 \mathrm{~nm}$ excitation and $580 \mathrm{~nm}$ emission for $45 \mathrm{~min}$ after an initial 5-minute baseline reading, with readings taken every $2 \mathrm{~min}$.

\subsection{Proliferation assay}

The effect of P2RX7B expression and inhibition on OS cell proliferation was assessed using the CellTiter $96^{\circledR}$ AQueous One Solution Cell Proliferation Assay (Promega, UK), TE85, TE85 + P2RX7B, MNNG-HOS and MNNG-HOS + P2RX7B cell lines were seeded at 5,000 and 2,500 cells per well, respectively, in a 96-well plate in $100 \mu \mathrm{L}$ phenol-free DMEM (Life Technologies, UK) complete med- 
ium. After 24 h, cells were washed twice with PBS and the medium was changed to medium containing $0.5 \%$ FBS. At each time-point on days $0,1,3,5$ and 7 , MTS was diluted 1:5 in phenol-free DMEM with $100 \mu \mathrm{L}$ added directly making a total volume of $200 \mu \mathrm{L}$. The cells were incubated for $3 \mathrm{~h}$ at $37^{\circ} \mathrm{C}$. The absorbance was read at $490 \mathrm{~nm}$ using the SpectraMax M5e Microplate Reader (Molecular Devices, USA). For inhibition studies the same protocol was performed with $100 \mu \mathrm{M}$ A740004 added after $24 \mathrm{~h}$ with absorbance read at day 3 (based on Giuliani et al., 2014 where A740003 was shown to effect cell growth at this concentration [15]).

\subsection{Cell adhesion assay}

96-well plates coated with $50 \mu \mathrm{g} / \mathrm{mL}$ Type 1 rat tail collagen (Sigma, UK) were seeded with 7,500 cells per well in medium containing $0.5 \% \mathrm{FBS}$. After incubation for $4 \mathrm{~h}$ at $37^{\circ} \mathrm{C}$ the wells were washed 4 times with PBS to removed unattached cells. Cells were then lysed using $50 \mu \mathrm{L}$ lysis buffer $\left(20 \mathrm{mM}\right.$ tris, $0.05 \mathrm{M} \mathrm{MgCl}_{2}$ ) and Quant-iT ${ }^{\mathrm{TM}}$ PicoGreen ${ }^{\circledR}$ dsDNA Reagent (ThermoFisher Scientific, UK) was then added to detect the DNA of the lysed remaining cells. Fluorescence was detected and quantified at $485 \mathrm{~nm}$ excitation and $530 \mathrm{~nm}$ emission.

\subsection{Cell migration (scratch assay)}

12-well plates were seeded with 200,000 cells in complete medium and left overnight to form a confluent monolayer. The medium was then changed to complete medium containing $5 \mu \mathrm{g} /$ $\mathrm{mL}$ mitomycin $\mathrm{C}$ and cells incubated for $2 \mathrm{~h}$ at $37^{\circ} \mathrm{C}$. A scratch was then made using a $10 \mu \mathrm{L}$ pipette tip down the centre of the well. The wells were washed twice with PBS and fresh 0.5\% FBS medium added. Images were then taken every $2 \mathrm{~h}$ for a $24 \mathrm{~h}$ period. For studies activating the P2RX7B $10 \mu \mathrm{M}$ BzATP was used (based on previous studies affecting migration in other cell types at this concentration $[16,36]$ ) in low $0.5 \%$ FBS medium. Scratch assay images were analysed using the automated T-scratch software [37].

\subsection{Cell invasion}

Matrigel $(1.5 \mathrm{mg} / \mathrm{mL})$ was added to Corning ${ }^{\circledR}$ FluoroBlok inserts and left to set for $2 \mathrm{~h}$ at $37^{\circ} \mathrm{C}$. Cells were incubated with $5 \mu \mathrm{g} / \mathrm{mL}$ mitomycin C for $2 \mathrm{~h}$ before 100,000 cells were added to Matrigelcoated insert in serum free medium $+10 \mu \mathrm{M}$ BzATP (based on previous studies affecting invasion in other cell types at this concentration $[16,36]) .10 \%$ FBS was used as a chemoattractant in the lower chamber. After $24 \mathrm{~h}$ the transwell inserts were washed twice in PBS and stained with $5 \mu \mathrm{M}$ Calcein AM cell permeant dye for $30 \mathrm{~min}$ at $37{ }^{\circ} \mathrm{C}$. The inserts were then washed twice again in PBS, imaged and analysed using Image J [38].

\subsection{RNA extraction and end point $P C R$}

RNA was extracted from cells using the ReliaPrep ${ }^{\mathrm{TM}}$ RNA Miniprep System kit in accordance with the manufacturer's protocol (Promega, UK). RNA was reverse transcribed using the Applied Biosystems ${ }^{\mathrm{TM}}$ high capacity RNA to $\mathrm{CDNA}^{\mathrm{TM}}$ Kit in accordance with the manufacturer's protocol. End point PCR primers were designed using Pubmed gene sequences. P2RX7 mRNA expression was determined with two sets of primers; the first primer set was designed in the $\mathrm{N}$ terminal gene sequence with the forward primer on the exon boundary between exon 3 and 4 and the reverse between exon 7 and 8 . This region is present on both the full length and truncated P2RX7B (Forward: 5'-TTGTGTCCCGAGTATCCCAC-3' Reverse: 5'-TCAATGCCCATTATTCCGCC-3' product length $413 \mathrm{bp}$,). The second primer set was designed further along the gene sequence with the both the forward primer and reverse primer designed to bind to exon 13 (Forward 5'-ACCAGAGGAGATA CAGCTGC-3' Reverse: 5'-TACTGCCCTTCACTCTTCGG-3', product length $399 \mathrm{bp}$ ). This C-terminal region is only present on the full length P2RX7A and thereby not detected in the cells with the truncated P2RX7B. cDNA was amplified using Promega GoTaq Flexi DNA polymerase kit with primers $(0.5 \mu \mathrm{M})$ and template cDNA added. The PCR samples were denatured for $2 \mathrm{~min}$ at $95{ }^{\circ} \mathrm{C}$ for one cycle, followed by 35 cycles of denaturation at $90{ }^{\circ} \mathrm{C}$ for $30 \mathrm{~s}$, primer annealing temperature for $30 \mathrm{~s}$, and extension at $72{ }^{\circ} \mathrm{C}$ for $30 \mathrm{~s}$. A final extension was then performed at $72{ }^{\circ} \mathrm{C}$ for $5 \mathrm{~min}$ for one cycle before samples were held at $4{ }^{\circ} \mathrm{C}$.

\subsection{Quantitative RT-PCR ( $q R T-P C R)$}

qRT-PCR was performed using Taqman probes (Human P2RX7 Taqman $^{\circledR}$ gene expression assay, ID: Hs00951600_m1 catalogue: 4351372, human HPRT Taqman $^{\circledR}$ gene expression assay, ID: Hs02800695 catalogue: 1621448) in accordance with the manufacturer's instructions on a Applied Biosystems 7900HT RealTime PCR machine (Applied Biosystems ${ }^{\mathrm{TM}}$ ).

2.10. RNA-seq of P2RX7B agonist and antagonist treated MNNG-HOS and MNNG-HOS + P2RX7B OS cell lines

MNNG-HOS and MNNG-HOS + P2RX7B cells were plated in a 6 well plate at a density of 500,000 cells and left for $24 \mathrm{~h}$ to adhere. Cells were then treated with $10 \mu \mathrm{M}$ BzATP, $10 \mu \mathrm{M}$ A740003 or both BzATP and A740003 together (A740003 was pre incubated for $1 \mathrm{~h}$ before adding BzATP), 24 h later RNA was extracted using the miRNeasy mini kit (Qiagen, UK) according to the manufacturer's protocol. RNA concentration and integrity were assessed on a NanoDrop 8000 Spectrophotometer (Thermo Fisher Scientific, UK) and RNA was stored at $-80^{\circ} \mathrm{C}$. The NEBNext ultra II RNA library prep kit (New England Biolabs, UK) was used to generate poly(A) + libraries. Sequencing was performed on a HiSeq 2500 (Illumina, USA) using a $150 \mathrm{bp}$ paired end (PE) metric.

\subsection{Bioinformatics}

Fastq files were converted to fasta. Trim Galore was used to remove adapter sequences and reads $<20$ nucleotides. Trimmed reads were aligned to the human genome (v38) using HISAT2 [39]. Transcripts were download from GENCODE (v28) and Ensembl (v92). Count matrices for transcripts were created using Kallisto [40]. Differentially expressed (DE) transcripts were determined using the DESeq2 [41] package in R (v1.2.10). DE mRNA according to $\log _{2}$ fold change $\geq 2, \mathrm{p} \leq 0.05$ plus false discovery rate $($ FDR $)<5 \%$ were selected for downstream analysis. Pathway analysis was used to determine the biological pathways of the different genes using the KEGG database.

\subsection{In silico analysis of OS datasets for P2RX7 expression}

A retrospective analysis across publicly available datasets was performed to identify P2RX7 expression in human OS cell lines. Searches were performed in the Cancer Dependency Map using the 20Q4 depmap gene expression dataset (Broad Institute, https://depmap.org/portal/), R2 Genomics Analysis and Visualization Platform using datasets (GSE86109, GSE42352, GSE11414, GSE124768 (http://r2.amc.nl) and Ordino a visual cancer analysis database https://ordino.caleydoapp.org/ where the gene P2RX7 was selected and filtered for OS, the identified cell lines were then selected for analysis. 


\subsection{In vivo animal studies}

All animal experiments were performed using 7-9-week-old female BALB/c nude mice (Charles River Margate, UK) that were acclimatised for at least one week prior to experimental manipulation. Mice were housed in the same environmentally controlled conditions with a $12 \mathrm{~h}$ light/dark cycle at $22{ }^{\circ} \mathrm{C}$ and free to access 2018 Teklad Global 18\% Protein Rodent Diet containing 1.01\% Calcium (Harlan Laboratories, UK) and water ad libitum in RB-3 cages. All procedures complied with the UK Animals (Scientific Procedures) Act 1986 and were reviewed and approved by the local Research Ethics Committee of The University of Sheffield (Sheffield, UK). Mice were anesthetized by inhalation of isoflurane and oxygen before injection of 250,000 MNNG-HOS or MNNGHOS + P2RX7B cells onto the tibial surface. For inhibition studies, mice were randomly allocated into treatment groups $(\mathrm{N}=11-13$ mice/group) 2 days after OS cell injection and received either vehicle (PBS + DMSO), or A740003 $(50 \mu \mathrm{g} / \mathrm{kg})$ by IP injection 3 times a week for up to 3 weeks. OS tumours were measured by callipers at the end of the experiment prior to euthanasia, the legs and lungs were then harvested for analysis. For OS metastasis studies mice were anesthetized as above before 1.5 million MNNG-HOS or MNNG-HOS + P2RX7B cells were injected into the tail vein. After 2 days the mice were divided into 4 groups ( $N=6$ mice/group) and treated with either vehicle (PBS + DMSO) or A740003 (50 $\mu \mathrm{g} /$ $\mathrm{kg}$ ) by IP injection 3 times a week for 3 weeks, after euthanasia the lungs were harvested.

\subsection{Micro-CT analysis}

Fixed tibiae were scanned using a SkyScan 1172 desktop $\mu \mathrm{CT}$ machine (Bruker) at a resolution of $8 \mu \mathrm{m}$ with the $\mathrm{X}$-ray source operating at $50 \mathrm{kV}, 200 \mu \mathrm{A}$ and using a $0.5 \mathrm{~mm}$ aluminium filter and images were captured every $0.7^{\circ}$. Scanned images were reconstructed using Skyscan NRecon software (v. 1.6.9, Bruker, Belgium). The region of interest (ROI) for the total bone volume was selected to include both the tibia and fibula and was determined at the top of the bone as soon as the tibia enters the image to the lower point where the tibia and fibula meet.

\subsection{Histological studies on the primary tumour}

Bones were fixed in neutral buffered formalin for $48 \mathrm{~h}$ after which time they were transferred to $70 \%$ ethanol. Bones were then decalcified in 10\% EDTA, embedded in paraffin and $5 \mu \mathrm{m}$ sections produced. Sections were then dewaxed in xylene, rehydrated through graded alcohols before performing heat mediated antigen retrieval using a water bath at $80{ }^{\circ} \mathrm{C}$ for $20 \mathrm{~min}$ with citrate buffer at pH6 (Abcam, Cambridge, UK). Endogenous peroxidase was blocked with $3 \%$ hydrogen peroxide (VWR, Lutterworth, UK) for 20 min at room temperature, washed twice in PBS-tween (PBST) and blocked in $1 \%$ Normal goat serum in PBST (Vector Laboratories, Peterborough, UK) for 20 min at room temperature. Primary rabbit anti-human Ki-67 antibody (Abcam $1 \mathrm{mg} / \mathrm{mL}$ ) was added to the sections at a dilution of 1:100 in $1 \%$ casein. Sections were incubated for $1 \mathrm{~h}$ at room temperature. After 2 washes in PBST, the sections were treated with biotinylated goat anti-rabbit IgG secondary antibody (Vector Laboratories) at 1:200 in 1\% casein for $20 \mathrm{~min}$ at room temperature. Sections were washed twice in PBST then treated with an $A B C$ kit (Vector Laboratories) for 20 min at room temperature and the bound antibody detected with Impact-DAB substrate-chromagen system (Vector Laboratories) for $5 \mathrm{~min}$ at room temperature. The sections were washed in tap water for $3 \mathrm{~min}$, counter stained in Gills haematoxylin (VWR, Merck, Birmingham, UK) for $5 \mathrm{~s}$, dehydrated through graded alcohols, and cleared with xylene. Coverslips were mounted using DPX. The slides were scanned using a Pannoramic 250 Flash III (3D HISTECH, Budapest, Hungary) and percentage of Ki-67 cells were quantified using QuPath software [42]. Tumours were further categorised histologically into high or low grade tumours using the median \% Ki67 value of $(20.17 \%)$ as a cut off [43].

\subsection{Histological studies for lung metastasis}

Lungs were collected and fixed in $10 \%$ neutral buffered formalin for $48 \mathrm{~h}$ before being changed into $70 \%$ ethanol and embedded in paraffin. To section the lungs $6 \mu \mathrm{m}$ sections were cut every $100 \mu \mathrm{m}$ deep to cover the entire lung. Sections were added to xylene twice for $5 \mathrm{~min}$ rehydrated through graded alcohols for $5 \mathrm{~min}$ each and tap water for $1 \mathrm{~min}$. The nuclei were then stained by Gill's II haematoxylin for $90 \mathrm{~s}$ and washed using tap water for 3 min. 1\% aqueous eosin (VWR, Merck, Birmingham, UK) with 1\% calcium carbonate (Sigma, UK) used to stain the cytoplasm for $5 \mathrm{~min}$. The slides were then quickly dehydrated through graded alcohols and cleared with xylene. Coverslips were mounted using DPX. Lung sections were visualised under a light microscope and were observed by two independent researchers blinded to the treatment groups for the presence of any OS metastatic nodules.

\subsection{Statistical analysis}

All data was analysed using GraphPad Prism v7. Datasets were tested for normality prior to analysis and statistical significance was tested using parametric or non-parametric tests as appropriate for the specific dataset. For comparing two groups, either a paired or unpaired $t$-test was used depending upon the groups to be compared. Two-way ANOVA was used to determine the effect of P2RX7B expression on tumour-induced changes in bone parameters in vivo. Slopes were compared using linear regression for the wound closure migration assay and a Chi-squared was used for Ki67 staining.

\section{Results}

\subsection{P2RX7B expression and functional characteristics in OS cells}

We have previously shown that the human OS cell line TE85 lacks endogenous P2RX7 protein expression [44] and that overexpression of the P2RX7 in this cell line confers trophic activity, with the most efficient growth-promoting isoform being P2RX7B alone [15]. To further investigate the role of the P2RX7B in OS pathology both TE85 and the MNNG-HOS cell line (a chemically transformed cell line derived from the original TE85 cell line which, unlike TE85 cells, forms tumours in mice in vivo [45]) were utilised. Similar to TE85 cells, we did not detect endogenous P2RX7 expression in MNNG-HOS cells at the mRNA transcript level using end point and qRT-PCR (Fig. 1A \& 1B). We also used the $\mathrm{Ca}^{2+}$ influx and pore formation assays to test for the presence of functional P2RX7, the results of which support the lack of endogenous P2RX7 in these cells (Fig. 1C \& 1D). We therefore transfected both these cell lines with the truncated P2RX7B isoform (Fig. 1A \& 1B), and confirmed functional expression using P2RX7 $\mathrm{Ca}^{2+}$ signalling and pore formation (Fig. 1C \& 1D), the latter being unaltered as predicated. To further examine the expression of P2RX7 in OS cell lines, we performed retrospective in silico analysis using publicly available datasets. DepMap contained P2RX7 gene expression data for 14 OS cell lines, R2 genomics had data for 38 cell lines (across 4 datasets) and Ordino had data for 9 cell lines. The data was then arranged showing P2RX7 expression across the cell lines. From these datasets the expression data for cell lines relating to the HOS-TE85 family and its modified derivatives KHOS (viral), MNNG 


\section{A)}

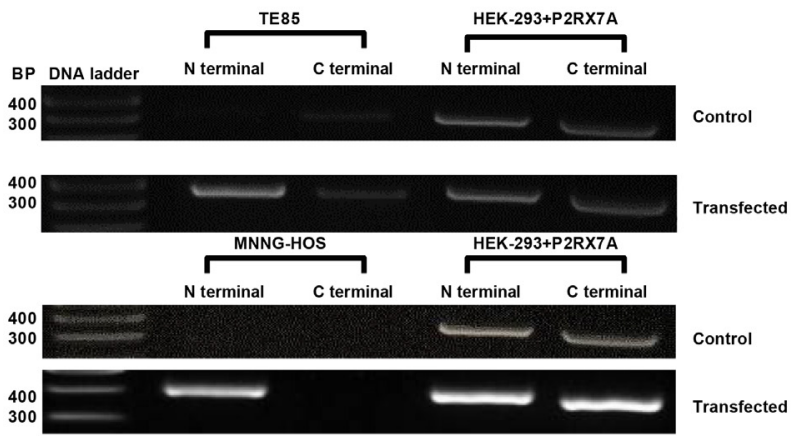

B)
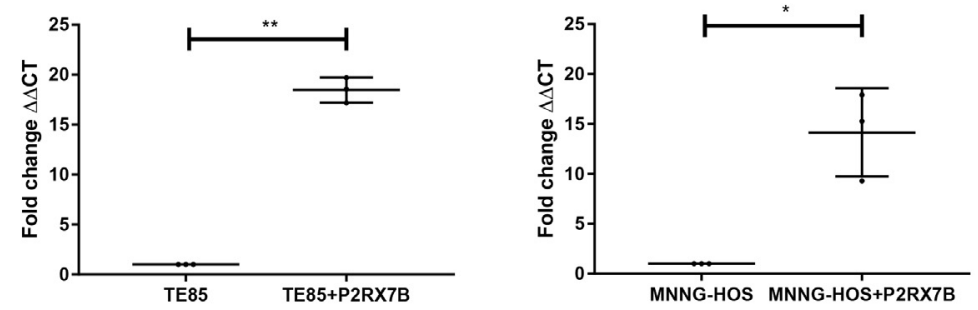

C)
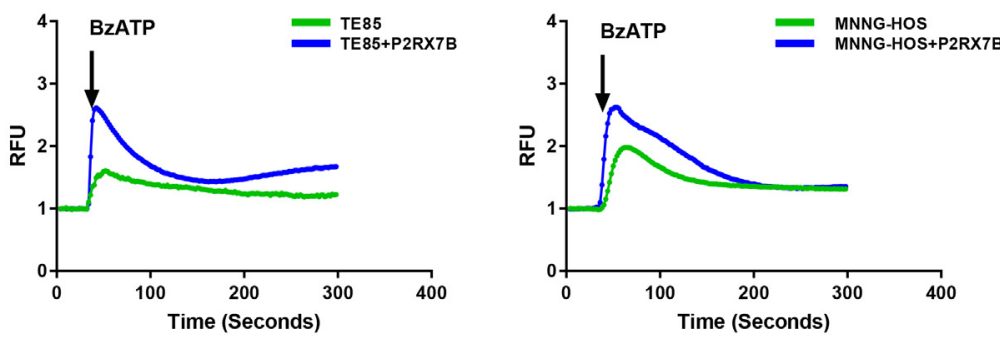

D)


HEK-293+P2RX7A
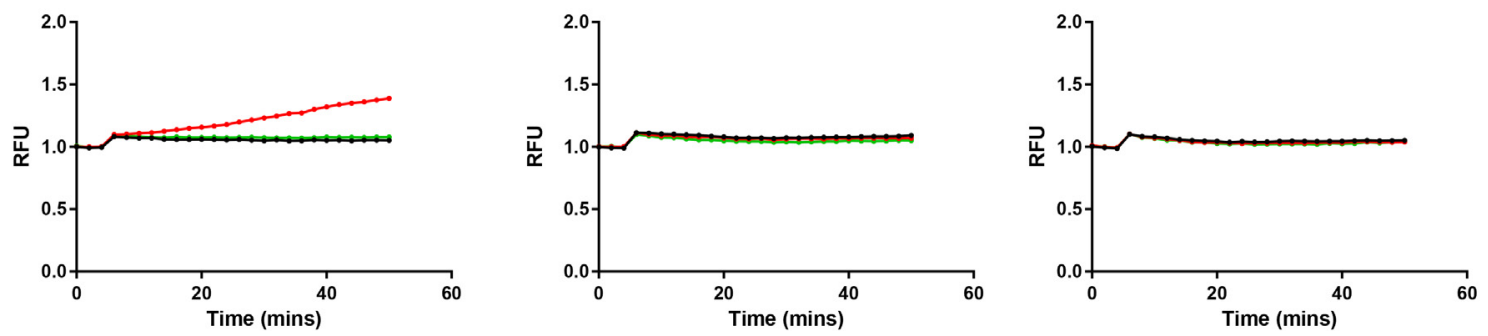


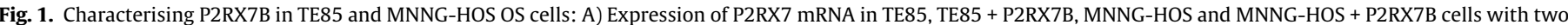

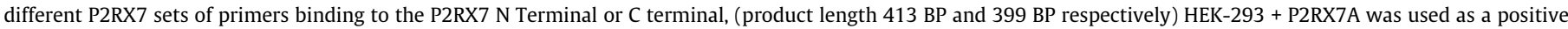

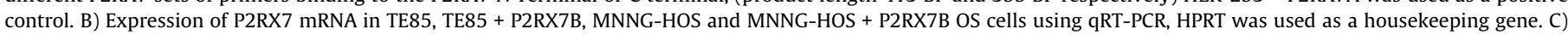





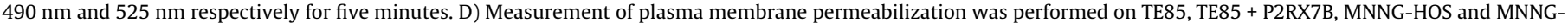

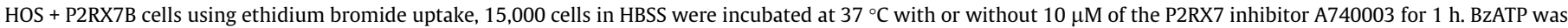

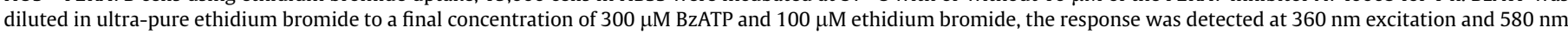

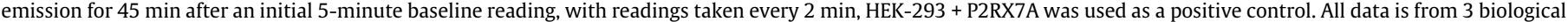
repeats with 6 technical replicates per experiment and were compared using an unpaired T-test, ${ }^{*}=\mathrm{P}<0.05^{* *}=\mathrm{P}<0.01$.

(chemical) and 143b (metastatic) [46,47] were consistently low for P2RX7 expression in comparison to other OS cell lines (Supplementary Fig. 1A-E). Cell lines that were consistently high for P2RX7 expression were SJSA-1 (Supplementary Fig. 1A-D), CAL72 (Supplementary Fig. 1B \& D) and MG63 (Supplementary Fig. 1A-D \& F).

\subsection{P2RX7B expression increases the growth and metastatic potential of OS cells}

Transfection of P2RX7B into both OS cell lines caused an increase in proliferation under low serum conditions over a 7day period. In TE85 cells transfected with P2RX7B $($ TE85 + P2RX7B) there was a growth increase of 33\% across day $3(\mathrm{P}=0.0001), 5(\mathrm{P}=0.0003)$ and $7(\mathrm{P}=0.0011)$ (Fig. 2Ai). The same increase in growth was also observed in MNNG-HOS cells transfected with P2RX7B (MNNG-HOS + P2RX7B) - 56\% increase across day $3(\mathrm{P}=<0.0001), 5(\mathrm{P}=<0.0001)$, and $7(\mathrm{P}=<0.0001)$ (Fig. 2Aiv). The P2RX7 antagonist A740003 reduced growth increase by $40 \%$ in TE85 + P2RX7B $(\mathrm{P}=<0.0001$, Fig. 2Aii $)$ and $52 \%$ in MNNGHOS + P2RX7B cells $(P=<0.0001$, Fig. 2Av). In both cell lines A740003 had no statistically significant effect on the parental cell lines not transfected with P2RX7B (Fig. 2Aiii and 2Avi).

To assess the effect of P2RX7B expression on adhesion, type 1 collagen was used as an extracellular matrix (ECM) and the cells ability to adhere to it was measured. TE85 + P2RX7B cells had a $47 \%$ reduction in cell adhesion $(\mathrm{P}=<0.0001$, Fig. $2 \mathrm{Bi})$ and MNNGHOS + P2RX7B cells had a $27 \%$ reduction in cell adhesion $(\mathrm{P}=<0.0001$, Fig. 2Bii) compared with their respective controls. To measure the effect of P2RX7B expression on OS cell migration a scratch assay was performed where the cells were monitored over a $24 \mathrm{~h}$ period for "wound" closure. After $24 \mathrm{~h}$ under low serum conditions, TE85 + P2RX7B showed a $12 \%$ increase in wound closure compared to TE85 cells and showed an overall difference when comparing the rate of closure ( $\mathrm{P}=<0.0001$, Fig. 2Ci and 2Ciii). This difference was further increased to $24 \%$ when stimulated with $10 \mu \mathrm{M}$ BzATP $(\mathrm{P}=<0.0001$, Fig. 2Civ). Similarly, MNNGHOS + P2RX7B cells showed a $15 \%$ increase in wound closure when stimulated with $10 \mu \mathrm{M}$ BzATP $(\mathrm{P}=0.002$, Fig. 2Cii and 2Cvi). To measure the invasion ability of OS cells a Fluroblok transwell system containing matrigel was used with $10 \%$ FBS in the lower chamber as a chemoattractant and the cells in the upper chamber stimulated with $10 \mu \mathrm{M}$ BzATP as P2RX7B receptor activation was required to induce increased migration in both OS cell lines. The TE85 cell line is a relatively non-invasive cell line therefore only a few detectable cells invaded the transwell (Fig. 2Di). Transfection with P2RX7B and stimulation with BzATP provided a strong stimulus to promote invasion increasing this from an average of 49 cells to 298 cells ( $\mathrm{P}=0.00374$, Fig. 2Dii). MNNG-HOS cells showed a higher level of invasion in keeping with their phenotype (Fig. 2Diii), with a similar increased invasion seen in the MNNGHOS + P2RX7B cells (increasing from an average of 278 cells to 662 cells, $P=0.025$, Fig. 2Div).

\subsection{Effect of $P 2 R X 7 B$ expression and inhibition on tumour growth and} bone disease in an OS mouse model in vivo

To determine whether P2RX7B expression would affect the growth and progression of OS disease in vivo a murine paratibial model of OS was used. This model has the advantage of an intact bone at the outset and results in tumours that reproduce the most common osteoblastic form of human OS [48-50]. As TE85 cells do not form tumours in vivo, and this remained the case even after transfection with P2RX7B (data not shown), we concentrated the in vivo part of this study using the MNNG-HOS cell lines. Injection of either MNNG-HOS or MNNG-HOS + P2RX7B cells resulted in the formation of OS in the tibia (Fig. 3A). There was no statistical difference in the final tumour size between mice injected with MNNGHOS + P2RX7B cells compared to MNNG-HOS cells $(\mathrm{P}=0.3071$, Supplementary Fig. 2Ai). The P2RX7 antagonist A740003 had a tendency to reduce the size of the tumours from both cell lines although this did not quite reach statistical significance ( $\mathrm{P}=0.0568$ for MNNG-HOS $+\mathrm{P} 2 \mathrm{RX7B}$ and $\mathrm{P}=0.0516$ for MNNGHOS; Supplementary Fig. 2Aii and 2Aiii). Ki-67 staining of the tumours similarly demonstrated no significant difference in the percentage of cells stained positive in MNNG-HOS + P2RX7B tumours compared to MNNG-HOS tumours ( $\mathrm{P}=0.6329$ Supplementary Fig. 2Bi) or when either group was treated with A740003 ( $P=0.9074$ Supplementary Fig. 2Ci and $P=0.4010$ respectively, Supplementary Fig. 2Di). When divided into high and low grade tumours, there was no difference in the percentage of grades between the cell lines ( $P=0.4201$ Supplementary Fig. 3Bii).

When both cells lines were treated with the P2RX7 antagonist A740003, the ratio of low to high grade tumours had a tendency to improve in both cell lines, however the association was not statistically significant, $(\mathrm{P}=0.1156$ for MNNG-HOS and $\mathrm{P}=0.3917$ for MNNG-HOS + P2RX7B respectively Supplementary Fig. 2Cii \& 3Dii).

A defining feature of OS is the involvement of the bone itself, therefore resultant effect on bone was measured using micro-CT. Injection of OS cells led to the typical ectopic bone formation (Fig. 3A) with a significant increase in total bone volume of the injected leg compared to its contralateral leg for both MNNGHOS $(P=0.0343$, Fig. 3Bi $)$ and MNNG-HOS + P2RX7B cells ( $P=0.0446$, Fig. 3Bii). When considering the effect of the cell type of OS tumour on the bone disease, Two-way ANOVA revealed that whilst the OS tumour accounted for $7.758 \%$ of the variance $(\mathrm{P}=0.0341)$, expression of $\mathrm{P} 2 \mathrm{RX} 7 \mathrm{~B}$ in the MNNG-HOS cells accounted for $13.62 \%$ of the variance $(P=0.0058)$. Treatment with A740003 reduced the extent of the tumour induced bone disease both in MNNG-HOS and MNNG-HOS + P2RX7B such that the bone volume was no longer significantly increased compared to the contralateral leg $(\mathrm{P}=0.2939$, and $\mathrm{P}=0.4783$ respectively, Fig. $3 \mathrm{~B})$.

\subsection{P2RX7B expression increases spontaneous OS lung metastasis in vivo}

To investigate the role of P2RX7B in OS metastasis the lungs were collected from each mouse from the paratibial model and 

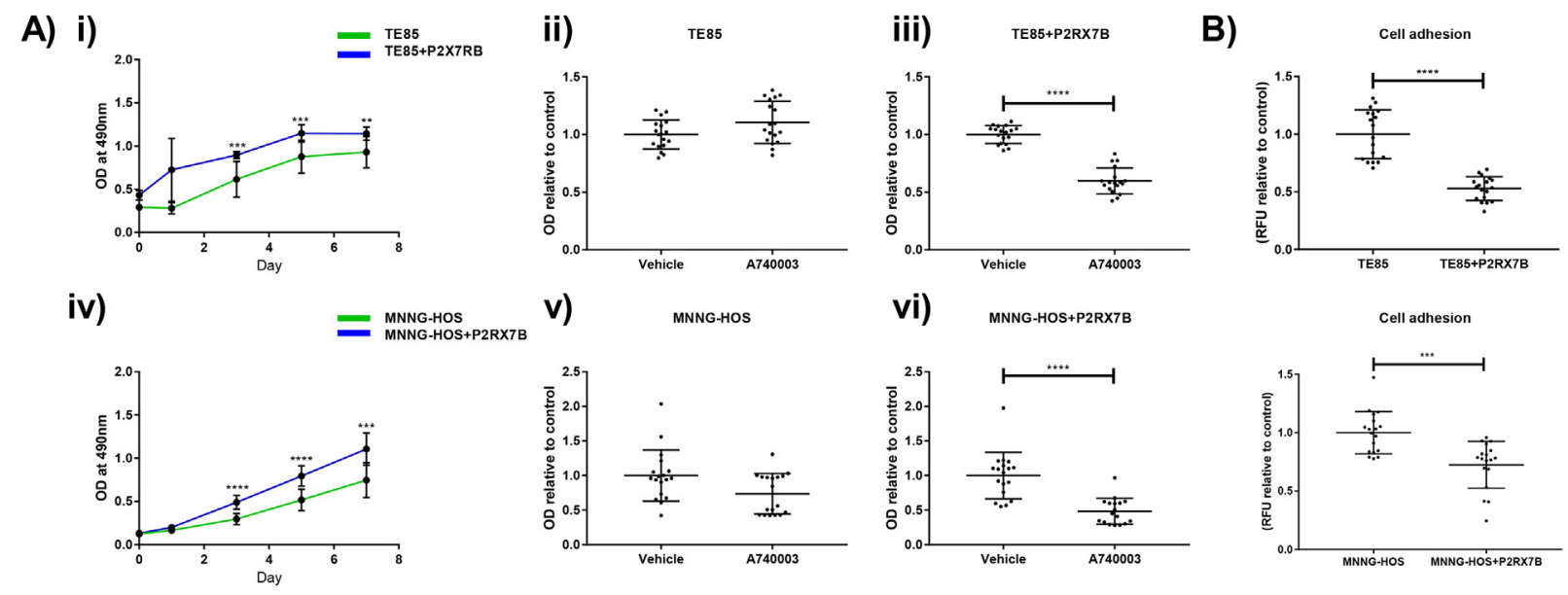

vi) MNNG-HOS+P2RX7B

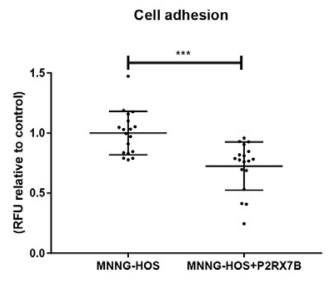

C) i)


iii)

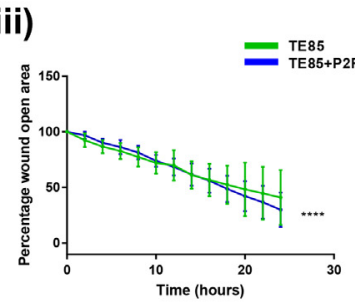

D) i)

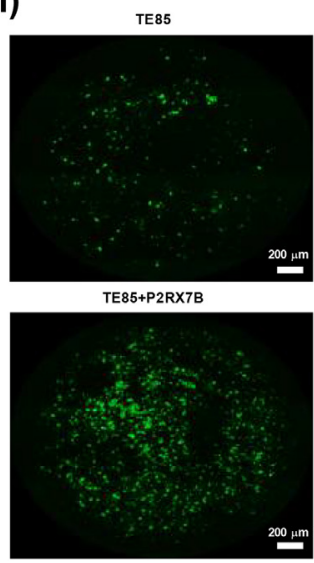

TE $85+P 2 R X 7 B$



iv)

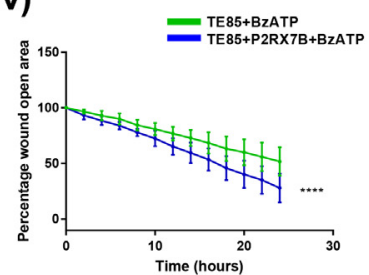

ii)



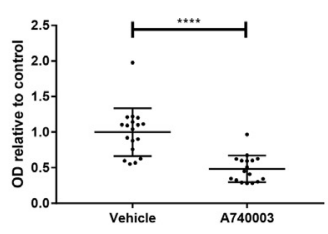

ii)

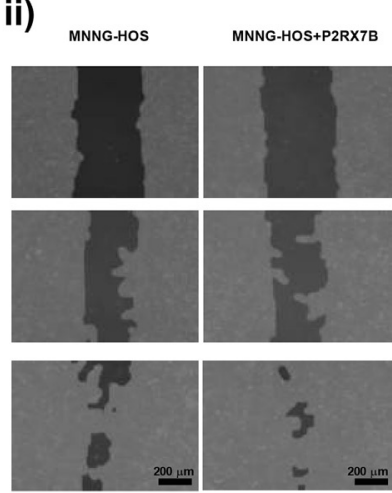

v)

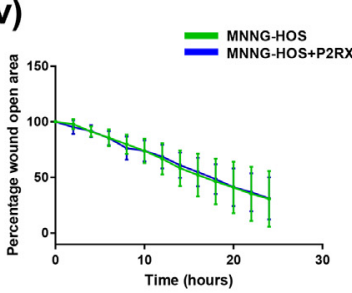

iii)



MNNG-HOS+BZATP MNNG-HOS+P2RX7B Time (hours)


12

vi)

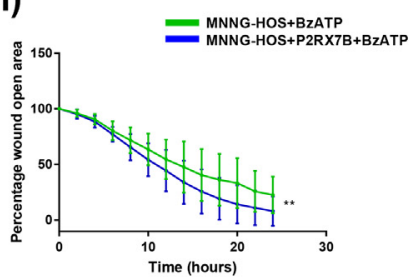

iv)

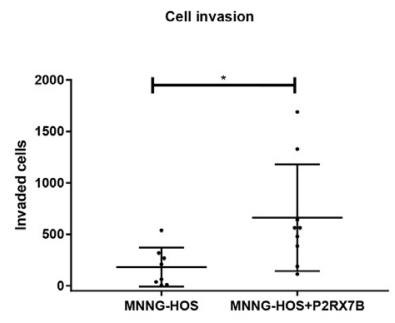




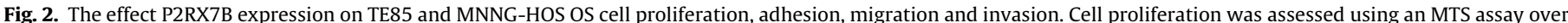

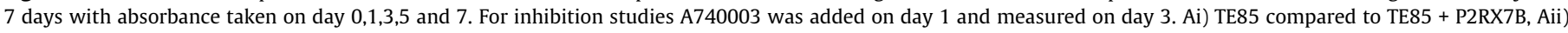



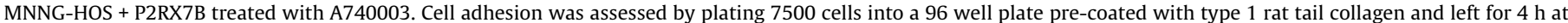

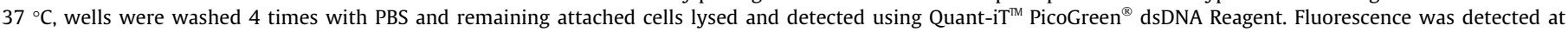

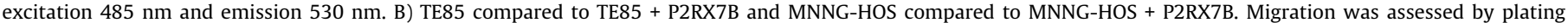

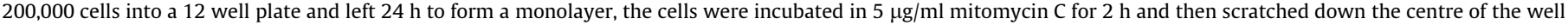

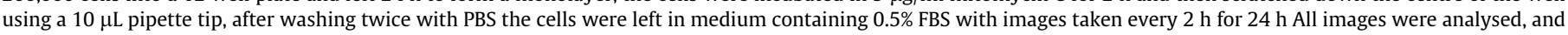

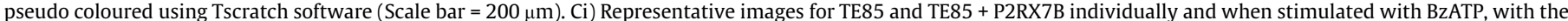

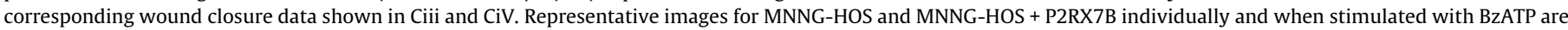

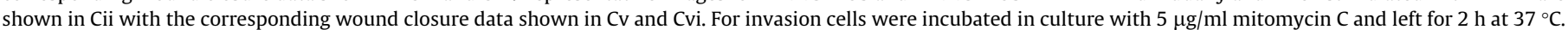

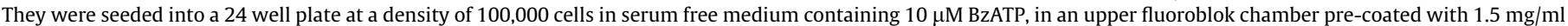



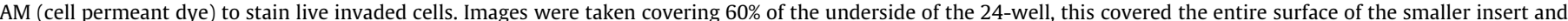

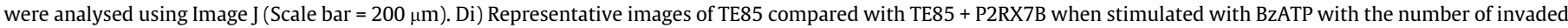



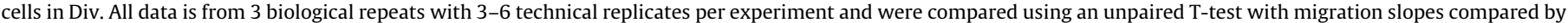
linear regression $^{*}=\mathrm{P}<0.05^{* *}=\mathrm{P}<0.01^{* * *}=\mathrm{P}<0.001^{* * * *}=\mathrm{P}<0.0001$.

examined histologically for signs of micrometastasis. We did not detect metastasis in any of the 26 mice bearing MNNG-HOS primary OS tumours, but we did detect metastasis in mice bearing MNNG-HOS + P2RX7B primary OS tumours. Out of 24 mice with primary OS tumours, 5 (21\%) had incidence of metastasis representing a significant difference $(\mathrm{p}=0.0142)$ in the incidence of detectable lung metastasis between the two cell lines (Fig. 4A \& $4 \mathrm{E})$. The P2RX7 antagonist had a tendency to reduce the incidence of metastasis from $3 / 13$ (23\%) mice for vehicle to $2 / 11$ (18\%) mice for A740003 (Fig. 4B \& 4E) but this did not reach statistical significance.

To further explore the role that P2RX7B plays in OS metastasis a lung colonization model was performed where mice were tail vein injected with either MNNG-HOS or MNNG-HOS + P2RX7B cells and treated with either vehicle or A740003. The incidence of lung metastasis using this model again tended to be higher in the mice
A) i)

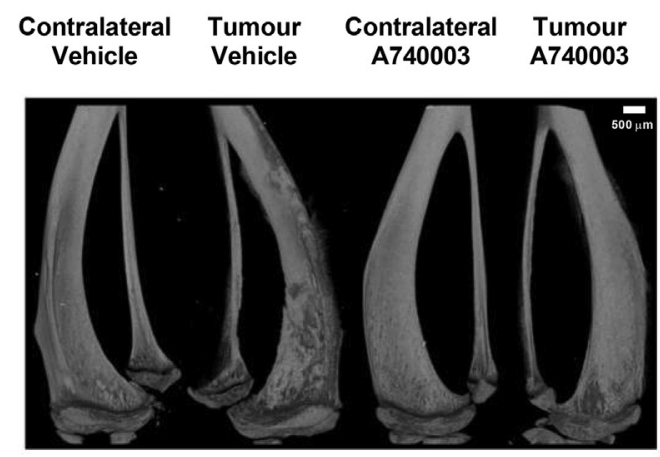

B) i) MNNG-HOS
Vehicle

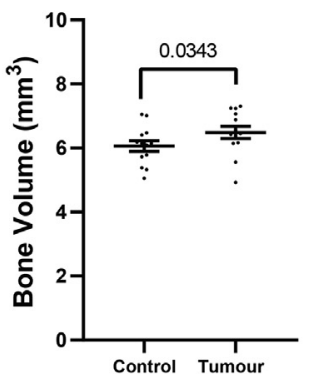

MNNG-HOS

A740003

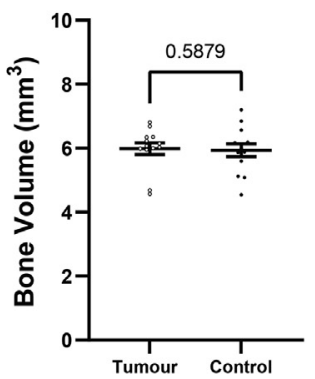

ii)

\section{MNNG-HOS+P2RX7B}

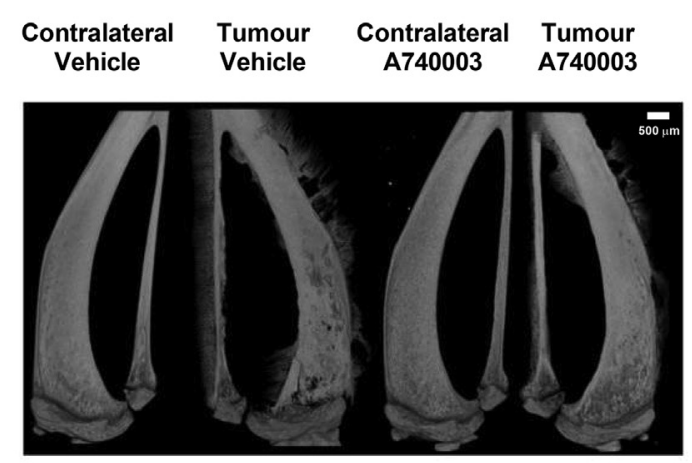

ii)
MNNG-HOS+P2RX7B A740003
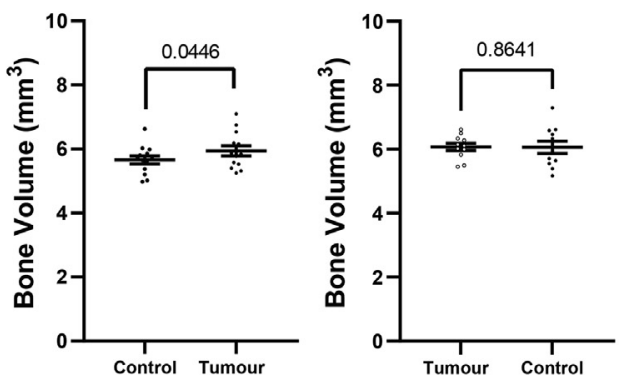

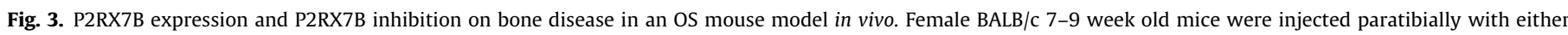

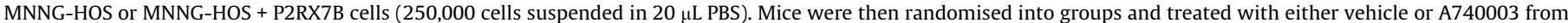

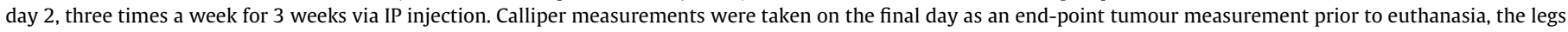

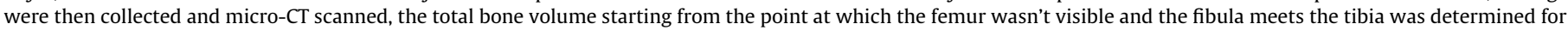



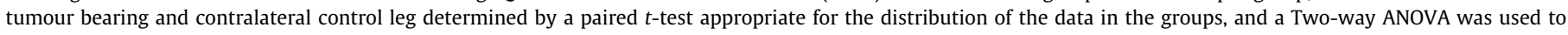

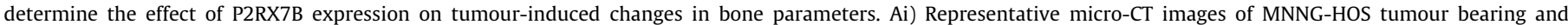

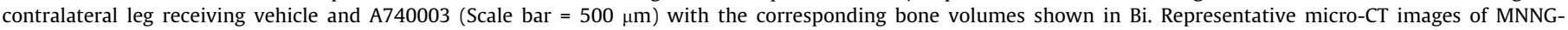
HOS + P2RX7B tumour bearing and contralateral leg receiving vehicle and A740003 are shown in Aii) and the corresponding bone volumes shown in Bii. 
A)

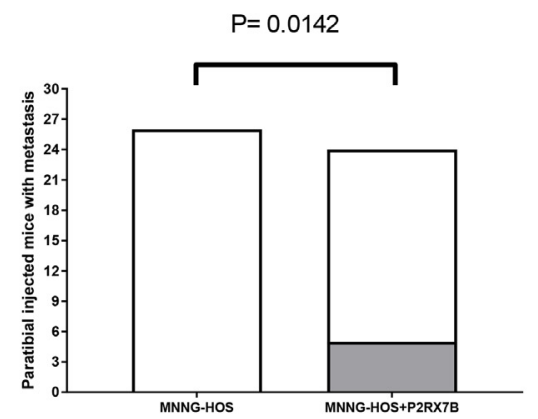

C)

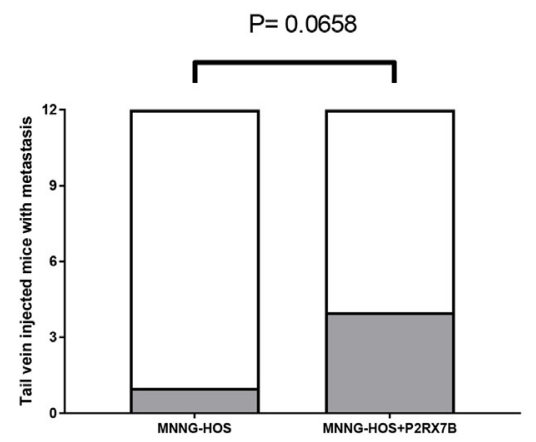

B)

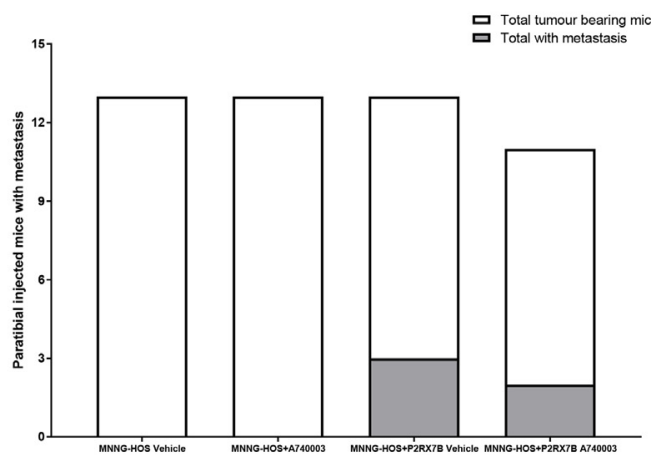

D)

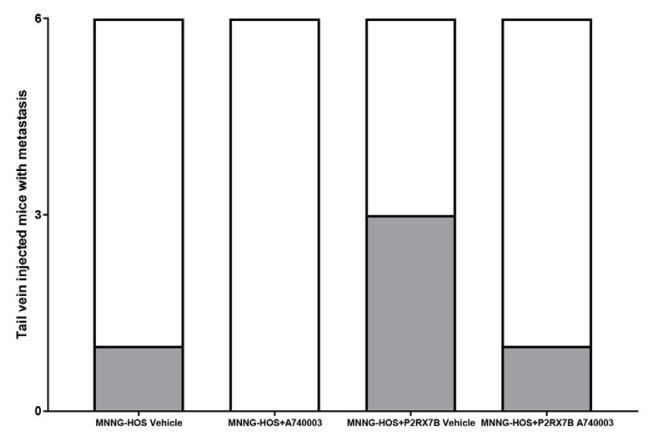

Paratibial injected

Tail vein injected

E)

\section{MNNG-HOS Vehicle}

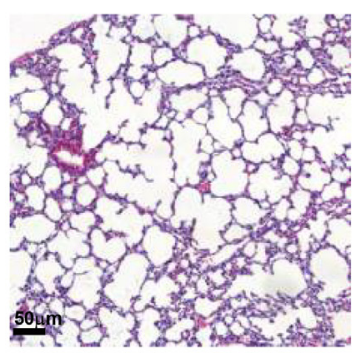

F)

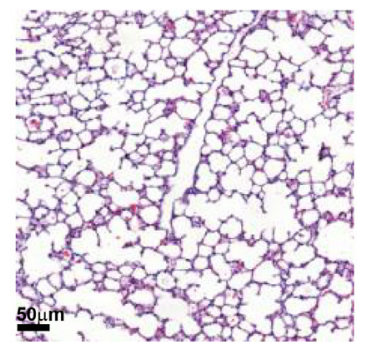

\section{MNNG-HOS} A740003

\section{MNNG-HOS A740003}

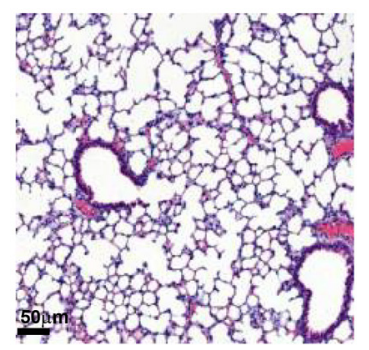

MNNG-HOS+P2RX7B MNNG-HOS+P2RX7B Vehicle

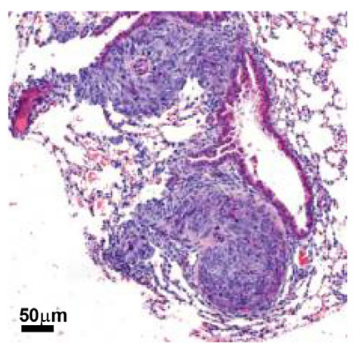

Paratibial injected


Tail vein injected

\section{MNNG-HOS+P2RX7B MNNG-HOS+P2RX7B A740003}


Fig. 4. P2RX7B expression increases OS metastasis in the lungs of MNNG-HOS and MNNG-HOS + P2RX7B paratibial and tail vein injected mice. Female BALB/c 7-9 week old mice were injected paratibially with 250,000 cells suspended in $20 \mu \mathrm{L}$ PBS of either MNNG-HOS or MNNG-HOS + P2RX7B cells. Mice were then randomised into groups and treated with either vehicle or A740003 from day 2, three times a week for 3 weeks via IP injection. For the tail vein model the same treatment regime was used after 1.5 million cells were injected. The mice were then euthanised and the lungs embedded into wax blocks sectioned and $6 \mu \mathrm{m}$ sections cut every $100 \mu \mathrm{m}$ deep to cover the entire lung. They were then stained using H\&E and visualised using a light microscope. A) Total number of OS lung metastasis in MNNG-HOS and MNNG-HOS + P2RX7B tumour bearing mice in the paratibial model. B) Number of OS lung metastasis in MNNG-HOS and MNNG-HOS + P2RX7B tumour bearing mice across treatment groups in the paratibial model. C) Total number of OS lung metastasis in MNNG-HOS and MNNG-HOS + P2RX7B injected mice in the tail vein model. D) Number of OS lung metastasis in MNNG-HOS and MNNG-HOS + P2RX7B injected mice across treatment groups in the tail vein model. E) Representative images of the lungs from MNNG-HOS and MNNGHOS + P2RX7B vehicle and A740003 treated in the paratibial model F) from MNNG-HOS and MNNG-HOS + P2RX7B vehicle and A740003 treated in the tail vein model (Scale bar $=50 \mu \mathrm{m})$.

injected with MNNG-HOS + P2RX7B cells (4/12 or 33\%) compared to the mice injected with MNNG-HOS (1/12 or 8\%) (Fig. 4C \& 4F,
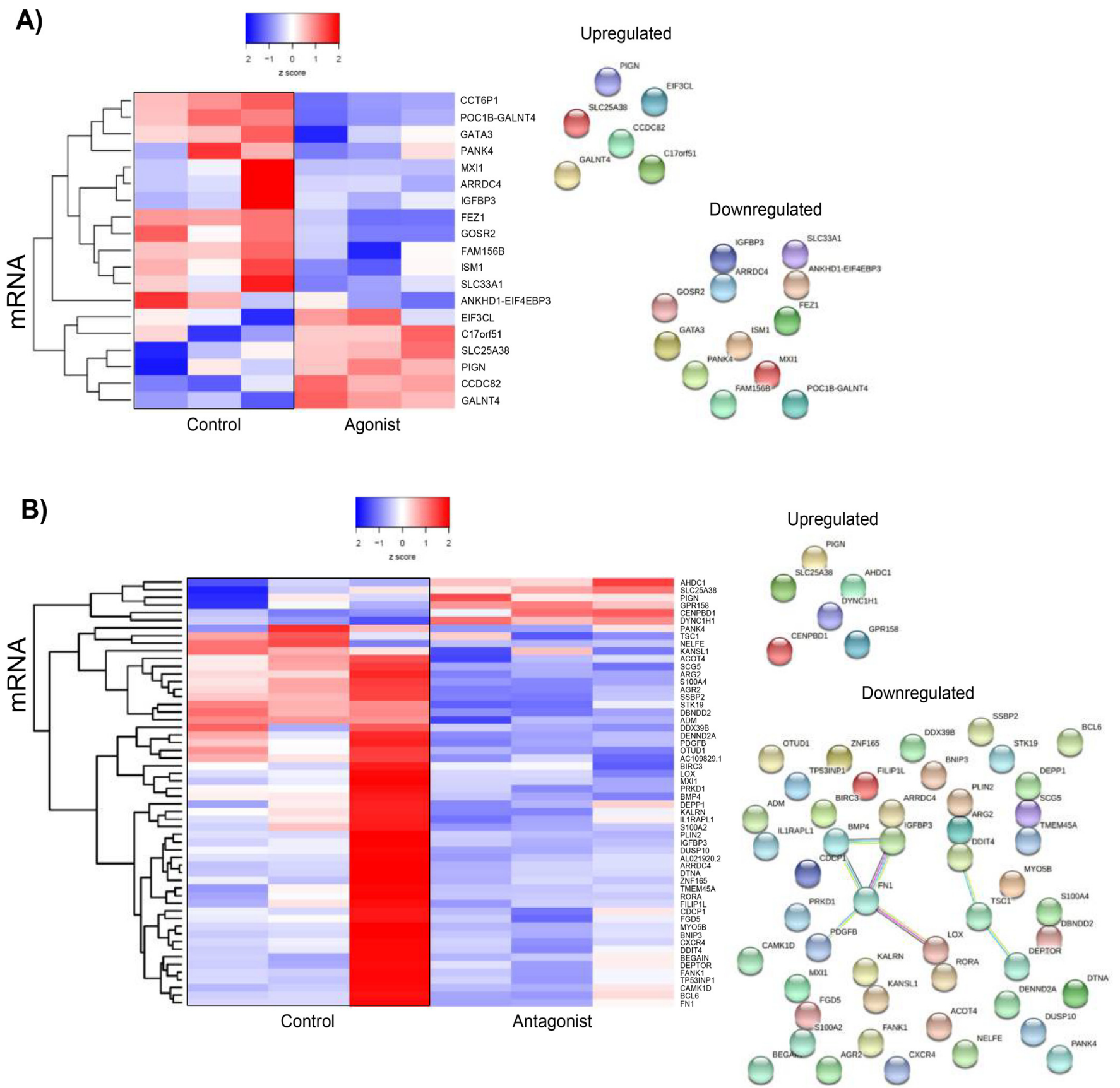

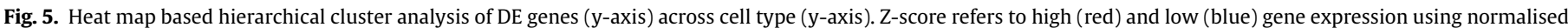

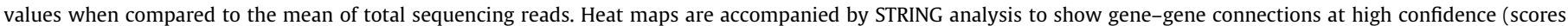

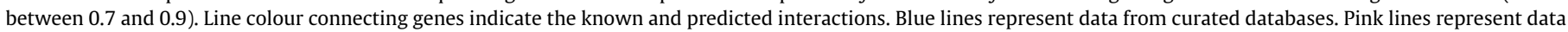

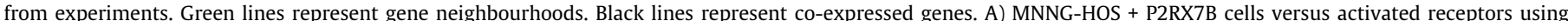

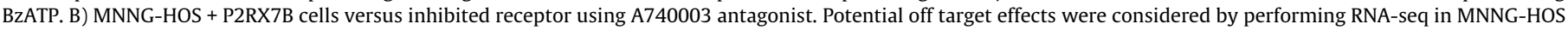
cells (Supplementary File). Each transcript presented has passed $\log _{2}$ fold change $\geq 2, p \leq 0.05$ and FDR $\leq 5 \%$ parameters. 
$\mathrm{p}=0.0658$ ). Treatment with the P2RX7 antagonist tended to reduce the incidence of metastasis to none in the MNNG-HOS cell injected mice, and from $3 / 6(50 \%)$ to $1 / 6(\sim 17 \%)$ for MNNNGHOS + P2RX7B injected mice (Fig. 4D \& 4F), although this did not reach statistical significance.

\subsection{P2RX7B inhibition drives downregulation of FN1/LOX/PDGFB/ IGFBP3/BMP4 axis}

To determine the downstream pathways contributing to the observed effects of P2RX7B signalling in OS cells in vitro and in vivo, we took a hypothesis free approach using RNA-seq. We performed heat map based hierarchical cluster analysis on DE genes unique to MNNG-HOS + P2RX7B cells when either treated with BzATP (Fig. 5A) or the antagonist A740003 (Fig. 5B). These genes were cross-referenced with several control cell lines to ensure that our data was derived from P2RX7B activation/inhibition and not from off target effects (Supplementary File). P2RX7B activation and antagonism were shown to drive independent responses, with antagonism specifically demonstrating a more potent biological response when in the context of cancer, showing a visibly higher number of genes affected and these are strongly related to cancer progression (Fig. 5 A-B). STRING analysis of gene-gene connections at high confidence level revealed an axis of downregulated genes in the P2RX7B antagonist treated MNNG-HOS + P2RX7B cells (Fig. 5B). This analysis revealed that P2RX7B antagonism downregulated an FN1/LOX/PDGFB/IGFBP3/BMP4 axis, which contains genes that are all known cancer drivers when upregulated [51].

\section{Discussion}

Despite being the most common type of primary bone cancer affecting children and adolescents, treatment options for OS have remained stagnant for the last 40 years or more. The overall fiveyear survival for all OS patients is $60 \%$ largely due to the fact that many patients relapse from chemoresistance or have metastasis on diagnosis reducing the survival rate in these patients to around $20 \%$ [3]. Treatment options are brutal and life changing, with surgery often leading to permanent disability and chemotherapy having significant side effects including infertility, cardio-, nephroand hepatic- toxicity. More targeted treatment options are needed to effectively treat OS patients with fewer treatment side effects.

We and others have previously reported the role of P2RX7 purinergic signalling in both the bone microenvironment and OS $[8,9,15,44]$, and this particular receptor has been increasingly implicated in cancer progression in various cancer types [52]. In this study we have built upon these observations and detailed the role of the P2RX7B splice variant specifically in OS. Having previously demonstrated that the cell line TE85 does not express functional P2RX7 $[15,44]$ we chose to use this cell line as well as the sub-clone MNNG-HOS to investigate the effect of the splice variant P2RX7B alone on OS cell behaviour. Prior to transfecting these cells with the P2RX7B, we confirmed that neither cell line expressed P2RX7 at the mRNA level. This finding is in direct contrast to a recent study, published during our own ongoing studies, which suggested that MNNG-HOS cells highly express P2RX7 [30]. We also scrutinised publicly available datasets to determine the relative levels of P2RX7 in the HOS family of OS cell lines and found that HOS, MNNG-HOS, KHOS and 143B cells all had low or nonexistent expression of P2RX7, further confirming our observations.

Following transfection of P2RX7B into TE85 and MNNG-HOS cells, end point PCR and qRT-PCR confirmed robust mRNA expression. There was also a substantially increased calcium response in P2RX7B transfected clones when stimulated with BzATP. Control cells also gave a slight increase in calcium response upon BzATP treatment, however this was not to the same extent as in
P2RX7B and may be due to activation of other BzATP-sensitive $\mathrm{P} 2$ receptors [53]. We also checked BzATP-induced pore formation in these cells, as despite P2RX7B not having the typical pore formation response due to a truncated $C$ terminal region known to be responsible for the pore, we have previously demonstrated that co-expression of P2RX7A and P2RX7B does lead to pore formation [15]. This further demonstrates a lack of endogenous P2RX7A expression in these cells, that in our clones the calcium increase relates to P2RX7B and in these clones a "non-functional" phenotype is present [26].

We have previously shown that when the full length P2RX7A variant was expressed alone in TE85 cells a growth advantage was observed, yet when both the P2RX7A and the P2RX7B variant were expressed together, although this enabled the typical P2RX7 pore formation, there was no further increase in growth. However, the strongest growth advantage was observed when the P2RX7B variant alone was expressed, prompting us to further investigate this in this study [15].We confirmed a strong growth advantage when P2RX7B was expressed in TE85 as expected and also MNNG-HOS cells. This growth advantage was observed in low serum conditions without exogenous activation of the receptor. However, we have previously shown that in Te85 cells expressing P2RX7 variants the enhanced growth advantage was diminished in the presence of apyrase suggesting that there is basal extracellular release of ATP by these cells to a concentration capable of activating P2RX7B [15]. The finding that the P2RX7 antagonists A740003 could significantly reduce this growth advantage offers promise for inhibiting P2RX7B mediated effects.

We next sought to determine the effect of P2RX7B expression on the ability of OS cells to leave the primary tumour site, invade surrounding tissues and spread to distant sites to form metastasis - the so called "invasion-metastasis cascade" [54]. This is particularly pertinent in OS and other highly metastatic cancers, as the 5year survival rate is drastically reduced in patients with metastasis [3]. Using established in vitro techniques, we found that P2RX7B expression reduced the ability of OS cells to adhere to a collagen ECM. Non-cancerous cells are normally tightly adherent to their ECM and also to each other - changes in cell-to-cell and cell-tomatrix adhesion is the first fundamental step in the metastatic cascade. E-cadherin is known to be pivotal in the control of cancer cell adhesion and spread, and it has previously been demonstrated that PX7R activation downregulates E-cadherin in breast cancer cells [55] Loss of E-cadherin mediated adhesion is also frequently associated with invasion and migration. Similar mechanisms involving cadherin signalling could be responsible for the P2RX7B-induced loss of adhesion in OS cells. Similar to the enhanced growth, activation of P2RX7B with the potent P2RX7 agonist BzATP, demonstrated increased invasion and migration rates in OS cells. Invasion requires the expression of various matrix metalloproteinases (MMPs) that are capable of digesting the ECM, allowing the cells to break away from the primary site and interact with tumour-associated stromal cells such as macrophages [51]. Activation of P2RX7 has been shown to induce the release of MMP13 in breast cancer cells [55] and has long been known to be responsible for the ATP-induced rapid release of MMP-9 from human peripheral-blood mononuclear cells [56]. This data further supports a pro-tumorigenic role of P2RX7 in OS as demonstrated previously $[15,30]$, but adds new knowledge that the $B$ variant alone plays a role in the metastasis cascade in OS cells in vitro.

Encouraged by the in vitro data, we next examined the role of P2RX7B in OS in vivo. As previously mentioned, the TE85 cell line does not produce tumours in mice therefore the MNNG-HOS cell lines only were used. Assessment of the growth of the primary tumour using calliper measurements and Ki-67 staining did not show any effect of P2RX7B expression or any effect of the P2RX7 antagonist A740003 on the final tumour volume or proliferation 
status. This was a surprising finding given the in vitro data and the previous study from Zhang et al. who showed a $50 \%$ reduction in the growth of intra-tibial MNNG-HOS tumours with A740003 treatment [30]. The differences between our data and theirs maybe explained by the different OS models used (paratibial versus intratibial injection of cells), timing of treatment ( 2 days post cell injection versus $\sim 10$ days) and also potential differences in the parental MNNG-HOS cell lines. As stated above we could not find any P2RX7 mRNA expression or typical function in the MNNG-HOS cells we used. When analysing the resultant bone disease in our OS model, expression of P2RX7B significantly affected the bone disease observed, with $A 740003$ reducing the extent of the tumour induced bone disease in both MNNG-HOS + P2RX7B and MNNGHOS such that the bone volume was no longer significantly increased compared to the contralateral leg. Whilst this is in keeping with the positive effect of P2RX7 antagonism on OS-induced bone disease previously reported, it is important to note again the difference between the two models. Here we show for the first time the specific involvement of P2RX7B in OS induced bone disease; that MNNG-HOS + P2RX7B cells produce an osteoblastic phenotype with formation of ectopic bone and so an increase in total bone volume which was reduced by treatment with A740003. Zhang et al show that the MNNG-HOS cells they used produced predominantly a lytic bone disease leading to reduced BV/TV which was also rescued with $A 740003$ treatment [30]. We also observed a reduction in the ectopic bone formation in the parental MNNG-HOS cells when treated with A740003. Considering that in our hands these cells do not express endogenous, functional P2RX7 receptors and the lack of effect on tumour size, the effect of A740003 may be mediated by the bone microenvironment itself, as opposed to a direct tumour driven effect. We and others have shown that both osteoblasts and osteoclasts express functional P2RX7 receptors [8], and the P2RX7 knockout mice display a bone phenotype [57-60]. Therefore treatment with A740003 could be affecting the resident bone cells and reducing the resultant bone disease in both subtypes of OS. Targeting the bone microenvironment in OS is a valid strategy, with other mediators of osteoblast and osteoclasts being previously proposed as potential therapeutic targets [61].

The five year survival rate for OS in the presence of secondary metastases is around $20 \%$. In addition, a quarter of patients presenting with detectable lung metastasis relapse. Gaining further insight into the potential mechanisms or predictors of OS metastasis is of paramount importance. By examining the lungs of the tumour bearing mice we found that P2RX7B expression in the primary tumour led to a significantly higher incidence of lung metastasis. This is in contrast to the lungs of the parental MNNG-HOS tumour bearing mice which showed no metastasis, in keeping with the previous observations that MNNG-HOS cells in vivo have no or very low incidence of pulmonary metastasis [45,47]. Taken together with the in vitro data of decreased adhesion with increased invasion and migration abilities of the MNNGHOS + P2RX7B cells and the minimal effect on tumour growth seen in our in vivo studies, these data support a role for the P2RX7B in the metastasis cascade in OS.

Previous studies in other cells types have elucidated various signalling pathways responsible for P2RX7 mediated effects such as via SK3 potassium channels and Cathepsin B [17], Cdc42 activity and the acquisition of a mesenchymal phenotype in breast cancer cells [21], Epithelial-mesenchymal transition (EMT) via SMAD2 phosphorylation in glioblastoma stem cells [19] and EMT/ invasion-related genes Snail, E-cadherin, Claudin-1, IL-8 and MMP-3 as well as PI3K/AKT and ERK1/2 in prostate cancer cells [18]. Whilst Zhang et al. also showed P2RX7 activation in OS cells promoted epithelial to mesenchymal transition and activated PI3K/Akt/GSK3 $\beta / \beta$-catenin and mTOR/HIF1 $\alpha /$ VEGF signalling [30].
Therefore, to try and elucidate further the pathways downstream of P2RX7B in OS we utilized a hypothesis-free RNA-seq approach. Using this methodology we revealed a set of genes that are differentially expressed upon both P2RX7B activation and antagonism. Antagonism of P2RX7B showed a more potent response in the context of cancer leading to a higher number of genes affected, but also suggesting that P2RX7B has activation state-dependent roles. These genes were strongly related to cancer and STRING analysis revealed a unique axis of downregulated genes in the antagonist treated MNNG-HOS + P2RX7B cells. Within this axis the genes FN1, LOX, PDGFB, IGFBP3 and BMP4 are known to promote cancer progression when upregulated in various cancer types. Roles for these genes/gene products in OS have also been reported: FN1 was shown to be upregulated in chemoresistant OS cells, was related to unfavourable prognosis and its inhibition greatly increased the sensitivity of OS cells to doxorubicin in vitro and in vivo [62]. LOX (Lysyl Oxidase) has been suggested to be tumour suppressor in OS [63], however we and others have shown that it is linked to hypoxia and plays a pro-tumorigenic role in cancers that metastasis to bone, inducing the pre-metastatic niche both in soft tissues such as lungs and in bone via formation of osteolytic lesion [64]. PDGFB is also linked to hypoxia and has been shown to be involved in OS cell proliferation and migration [65], and IGFBP3 has also been shown to stimulate OS migration [66]. The BMP4 protein, part of the bone morphogenetic family which are known critical regulators of bone formation and remodelling, has recently been shown to be the most frequently expressed BMP in musculoskeletal tumours, including OS, in a recent study of 1,361 patients with 22 types of musculoskeletal tumours [67]. Elucidating the interplay between P2RX7B and these signalling pathways could reveal common targets at which they converge and that may prove to be fundamental in OS progression and metastasis. Further studies should look to determine the effect of combination treatments (both within this axis and with current OS drugs) that may prove to be more efficacious than single agent treatments. Combining treatments for signalling pathways specifically involved in OS would lead to kinder, less harsh treatments for the patient, fewer relapses and a better overall prognosis.

\section{Conclusion}

In summary we present data for the first time that the specific P2RX7B isoform, which has a distinct non-functional phenotype compared with full length P2RX7, is associated with OS progression, bone disease and metastasis. Future studies exploring the therapeutic targeting of P2RX7 need to pay particular attention to the isoforms being targeted as well as the OS subtype.

\section{Declarations}

Ethical Approval

All procedures complied with the UK Animals (Scientific Procedures) Act 1986 and were reviewed and approved by the local Research Ethics Committee of The University of Sheffield (Sheffield, UK)

\section{Consent for publication}

Not applicable

\section{Availability of data and materials}

The data that support the findings of this study are available on request from the corresponding author. Raw sequencing files are 
available at Sequence Read Archive (www.ncbi.nlm.nih.gov/sra) under the accession SRX10116510.

\section{Competing interests}

F.D.V. is a member of the Scientific Advisory Board of Biosceptre Ltd., a Biotech involved in the development of P2RX7-targeted therapies. The remaining authors declare no competing interests.

\section{CRediT authorship contribution statement}

Luke Tattersall: Data curation, Formal analysis, Investigation, Methodology, Writing - original draft. Karan M. Shah: Data curation, Formal analysis, Investigation, Methodology, Writing - review \& editing. Darren L. Lath: Methodology, Formal analysis, Writing review \& editing. Archana Singh: Methodology, Formal analysis, Writing - review \& editing. Jennifer M. Down: Methodology, Writing - review \& editing. Elena De Marchi: Methodology, Writing review \& editing. Alex Williamson: Methodology, Writing review \& editing. Francesco Di Virgilio:Resources, Writing review \& editing. Dominique Heymann: Resources, Conceptualization, Writing - review \& editing. Elena Adinolfi: Resources, Supervision, Writing - review \& editing. William D. Fraser: Resources, Writing - review \& editing. Darrell Green: Resources, Data curation, Formal analysis, Writing - review \& editing. Michelle A. Lawson: Conceptualization, Funding acquisition, Data curation, Formal analysis, Investigation, Methodology, Resources, Supervision, Writing - review \& editing. Alison Gartland: Conceptualization, Funding acquisition, Data curation, Formal analysis, Investigation, Methodology, Resources, Supervision, Writing review \& editing.

\section{Acknowledgements}

We would like to thank the Bone Cancer Research Trust for funding this work (Grant number 4415 awarded to A.G), and the Bone Analysis Laboratory within the Department of Oncology and Metabolism for technical assistance.

Appendix A. Supplementary data

Supplementary data to this article can be found online at https://doi.org/10.1016/j.jbo.2021.100398.

\section{References:}

[1] M.L. Broadhead, J.C.M. Clark, D.E. Myers, C.R. Dass, P.F.M. Choong, The Molecular Pathogenesis of Osteosarcoma : A Review, Sarcoma 2011 (2011) 959248.

[2] C. Gerrand, N. Athanasou, B. Brennan, R. Grimer, I. Judson, B. Morland, D. Peake, B. Seddon, J. Whelan, G., British Sarcoma, UK guidelines for the management of bone sarcomas, Clin Sarcoma Res 6 (2016) 7.

[3] D.C. Allison, S.C. Carney, E.R. Ahlmann, A. Hendifar, S. Chawla, A. Fedenko, C. Angeles, L.R. Menendez, A meta-analysis of osteosarcoma outcomes in the modern medical era, Sarcoma 2012 (2012) 704872.

[4] F. Di Virgilio, E. Adinolfi, Extracellular purines, purinergic receptors and tumor growth, Oncogene 36 (3) (2017) 293-303.

[5] E. De Marchi, E. Orioli, A. Pegoraro, E. Adinolfi, F. Di Virgilio, Detection of Extracellular ATP in the Tumor Microenvironment, Using the pmeLUC Biosensor, Methods Mol Biol 2041 (2020) 183-195.

[6] E. De Marchi, E. Orioli, A. Pegoraro, S. Sangaletti, P. Portararo, A. Curti, M.P. Colombo, F. Di Virgilio, E. Adinolfi, The P2X7 receptor modulates immune cells infiltration, ectonucleotidases expression and extracellular ATP levels in the tumor microenvironment, Oncogene 38 (19) (2019) 3636-3650.

[7] P. Pellegatti, L. Raffaghello, G. Bianchi, F. Piccardi, V. Pistoia, F. Di Virgilio, J. El Khoury, Increased level of extracellular ATP at tumor sites: in vivo imaging with plasma membrane luciferase, PLoS One 3 (7) (2008) e2599.

[8] A. Agrawal, A. Gartland, P2X7 receptors: role in bone cell formation and function, J Mol Endocrinol 54 (2) (2015) R75-R88.

[9] R.M. Rumney, N. Wang, A. Agrawal, A. Gartland, Purinergic signalling in bone, Front Endocrinol (Lausanne) 3 (2012) 116

[10] D.C. Genetos, D.J. Geist, D. Liu, H.J. Donahue, R.L. Duncan, Fluid shear-induced ATP secretion mediates prostaglandin release in MC3T3-E1 osteoblasts, J Bone Miner Res 20 (1) (2005) 41-49.
[11] R.M.H. Rumney, A. Sunters, G.C. Reilly, A. Gartland, Application of multiple forms of mechanical loading to human osteoblasts reveals increased ATP release in response to fluid flow in 3D cultures and differential regulation of immediate early genes, J Biomech 45 (3) (2012) 549-554.

[12] D. Ferrari, F. Malavasi, L. Antonioli, A Purinergic Trail for Metastases, Trends Pharmacol Sci 38 (3) (2017) 277-290.

[13] E. Adinolfi, L. Raffaghello, A.L. Giuliani, L. Cavazzini, M. Capece, P. Chiozzi, G. Bianchi, G. Kroemer, V. Pistoia, F. Di Virgilio, Expression of P2X7 receptor increases in vivo tumor growth, Cancer Res 72 (12) (2012) 2957-2969.

[14] F. Amoroso, M. Capece, A. Rotondo, D. Cangelosi, M. Ferracin, A. Franceschini, L. Raffaghello, V. Pistoia, L. Varesio, E. Adinolfi, The P2X7 receptor is a key modulator of the PI3K/GSK3beta/VEGF signaling network: evidence in experimental neuroblastoma, Oncogene 34 (41) (2015) 5240-5251.

[15] A.L. Giuliani, D. Colognesi, T. Ricco, C. Roncato, M. Capece, F. Amoroso, Q.G. Wang, E. De Marchi, A. Gartland, F. Di Virgilio, E. Adinolfi, J. Kanellopoulos, Trophic activity of human P2X7 receptor isoforms A and B in osteosarcoma, PLoS One 9 (9) (2014) e107224.

[16] A. Giannuzzo, M. Saccomano, J. Napp, M. Ellegaard, F. Alves, I. Novak, Targeting of the P2X7 receptor in pancreatic cancer and stellate cells, Int J Cancer 139 (11) (2016) 2540-2552.

[17] B. Jelassi, A. Chantôme, F. Alcaraz-Pérez, A. Baroja-Mazo, M.L. Cayuela, P. Pelegrin, A. Surprenant, S. Roger, P2X(7) receptor activation enhances SK3 channels- and cystein cathepsin-dependent cancer cells invasiveness, Oncogene 30 (18) (2011) 2108-2122.

[18] Y. Qiu, W.-hua. Li, H.-quan. Zhang, Y. Liu, X.-X. Tian, W.-G. Fang, J. Kanellopoulos, P2X7 mediates ATP-driven invasiveness in prostate cancer cells, PLoS One 9 (12) (2014) e114371.

[19] S. Ziberi, M. Zuccarini, M. Carluccio, P. Giuliani, L. Ricci-Vitiani, R. Pallini, F. Caciagli, P. Di Iorio, R. Ciccarelli, Upregulation of Epithelial-To-Mesenchymal Transition Markers and P2X7 Receptors Is Associated to Increased Invasiveness Caused by P2X7 Receptor Stimulation in Human Glioblastoma Stem Cells, Cells 9 (1) (2019) 85, https://doi.org/10.3390/cells9010085.

[20] Y. Zhang, F. Li, L. Wang, Y. Lou, A438079 affects colorectal cancer cell proliferation, migration, apoptosis, and pyroptosis by inhibiting the P2X7 receptor, Biochem Biophys Res Commun 558 (2021) 147-153.

[21] L. Brisson, S. Chadet, O. Lopez-Charcas, B. Jelassi, D. Ternant, J. Chamouton, S. Lerondel, A. Le Pape, I. Couillin, A. Gombault, F. Trovero, S. Chevalier, P. Besson, L.-H. Jiang, Sébastien Roger, P2X7 Receptor Promotes Mouse Mammary Cancer Cell Invasiveness and Tumour Progression, and Is a Target for Anticancer Treatment, Cancers (Basel) 12 (9) (2020) 2342, https://doi.org/ $10.3390 /$ cancers12092342.

[22] E. Adinolfi, M. Cirillo, R. Woltersdorf, S. Falzoni, P. Chiozzi, P. Pellegatti, M.G. Callegari, D. Sandonà, F. Markwardt, Günther Schmalzing, F. Di Virgilio, Trophic activity of a naturally occurring truncated isoform of the P2X7 receptor, FASEB J 24 (9) (2010) 3393-3404.

[23] B. Cheewatrakoolpong, H. Gilchrest, J.C. Anthes, S. Greenfeder, Identification and characterization of splice variants of the human P2X7 ATP channel, Biochem Biophys Res Commun 332 (1) (2005) 17-27.

[24] Y.-H. Feng, X. Li, L. Wang, L. Zhou, G.I. Gorodeski, A truncated P2X7 receptor variant (P2X7-j) endogenously expressed in cervical cancer cells antagonizes the full-length P2X7 receptor through hetero-oligomerization, J Biol Chem 281 (25) (2006) 17228-17237.

[25] R. Sluyter, L. Stokes, Significance of P2X7 receptor variants to human health and disease, Recent Pat DNA Gene Seq 5 (1) (2011) 41-54.

[26] SM Gilbert, CJ Oliphant, S. Hassan, AL Peille, P. Bronsert, S. Falzoni, F. Di Virgilio, S. McNulty, R. Lara, ATP in the tumour microenvironment drives expression of nfP2X7, a key mediator of cancer cell survival, Oncogene 38 (2) (2019) 194-208.

[27] A. Pegoraro, E. De Marchi, E. Adinolfi, P2X7 Variants in Oncogenesis, Cells 10 (1) (2021) 189, https://doi.org/10.3390/cells10010189.

[28] J. Benzaquen, S.J. Dit Hreich, S. Heeke, T. Juhel, S. Lalvee, S. Bauwens, S. Saccani, P. Lenormand, Véronique Hofman, M. Butori, S. Leroy, J.-P. Berthet, C.-H. Marquette, P. Hofman, V. Vouret-Craviari, P2RX7B is a new theranostic marker for lung adenocarcinoma patients, Theranostics 10 (24) (2020) $10849-10860$.

[29] A. Pegoraro, E. Orioli, E. De Marchi, V. Salvestrini, A. Milani, F. Di Virgilio, A. Curti, E. Adinolfi, Differential sensitivity of acute myeloid leukemia cells to daunorubicin depends on P2X7A versus P2X7B receptor expression, Cell Death Dis 11 (10) (2020) 876.

[30] Y. Zhang, H. Cheng, W. Li, H. Wu, Y. Yang, Highly-expressed P2X7 receptor promotes growth and metastasis of human HOS/MNNG osteosarcoma cells via PI3K/Akt/GSK3beta/beta-catenin and mTOR/HIF1alpha/VEGF signaling, Int J Cancer 145 (4) (2019) 1068-1082.

[31] R.M. McAllister, M.B. Gardner, A.E. Greene, C. Bradt, W.W. Nichols, B.H. Landing, Cultivation in vitro of cells derived from a human osteosarcoma, Cancer 27 (2) (1971) 397-402.

[32] J.S. Rhim, D.L. Putman, P. Arnstein, R.J. Huebner, R.M. McAllister, Characterization of human cells transformed in vitro by N-methyl-N'-nitroN-nitrosoguanidine, Int J Cancer 19 (4) (1977) 505-510.

[33] P.E. Mains, I.A. Sulston, W.B. Wood, Dominant maternal-effect mutations causing embryonic lethality in Caenorhabditis elegans, Genetics 125 (2)(1990) 351-369.

[34] A. Agrawal, L. S. Kruse, A. J. Vangsted, A. Gartland, N. R. Jørgensen, Human P2X7 Receptor Causes Cycle Arrest in RPMI-8226 Myeloma Cells to Alter the Interaction with Osteoblasts and Osteoclasts, Cells 9 (11) (2020) 2341, https:// doi.org/10.3390/cells9112341. 
[35] A.W. Farrell, S. Gadeock, A. Pupovac, B. Wang, I. Jalilian, M. Ranson, R. Sluyter, P2X7 receptor activation induces cell death and CD23 shedding in human RPMI 8226 multiple myeloma cells, Biochim Biophys Acta 1800 (11) (2010) 1173-1182.

[36] A. Giannuzzo, S.F. Pedersen, I. Novak, The P2X7 receptor regulates cell survival, migration and invasion of pancreatic ductal adenocarcinoma cells, Mol Cancer 14 (2015) 203.

[37] T. Geback, M.M. Schulz, P. Koumoutsakos, M. Detmar, TScratch: a novel and simple software tool for automated analysis of monolayer wound healing assays, Biotechniques 46 (4) (2009) 265-274.

[38] C.T. Rueden, J. Schindelin, M.C. Hiner, B.E. DeZonia, A.E. Walter, E.T. Arena, K.W. Eliceiri, Image J2: Image for the next generation of scientific image data, BMC Bioinformatics 18 (1) (2017) 529.

[39] M. Pertea, D. Kim, G.M. Pertea, J.T. Leek, S.L. Salzberg, Transcriptlevel expression analysis of RNA-seq experiments with HISAT, StringTie and Ballgown, Nat Protoc 11 (9) (2016) 1650-1667.

[40] N.L. Bray, H. Pimentel, Páll Melsted, L. Pachter, Near-optimal probabilistic RNA-seq quantification, Nat Biotechnol 34 (5) (2016) 525-527.

[41] M.I. Love, W. Huber, S. Anders, Moderated estimation of fold change and dispersion for RNA-seq data with DESeq2, Genome Biol 15 (12) (2014) 550.

[42] P. Bankhead, M.B. Loughrey, J.A. Fernandez, Y. Dombrowski, D.G. McArt, P.D. Dunne, S. McQuaid, R.T. Gray, L.J. Murray, H.G. Coleman, J.A. James, M. SaltoTellez, P.W. Hamilton, QuPath: Open source software for digital pathology image analysis, Sci Rep 7 (1) (2017) 16878.

[43] K. Scotlandi, M. Serra, M.C. Manara, D. Maurici, S. Benini, G. Nini, M. Campanacci, N. Baldini, Clinical relevance of Ki-67 expression in bone tumors, Cancer 75 (3) (1995) 806-814.

[44] A. Gartland, R.A. Hipskind, J.A. Gallagher, W.B. Bowler, Expression of a P2X7 receptor by a subpopulation of human osteoblasts, J Bone Miner Res 16 (5) (2001) 846-856.

[45] A.B. Mohseny, I. Machado, Y. Cai, K.-L. Schaefer, M. Serra, P.C.W. Hogendoorn, A. Llombart-Bosch, A.-M. Cleton-Jansen, Functional characterization of osteosarcoma cell lines provides representative models to study the human disease, Laboratory Investigation 91 (8) (2011) 1195-1205.

[46] T. Ikeda, Y. Futaesaku, N. Tsuchida, In vitro differentiation of the human osteosarcoma cell lines, HOS and KHOS, Virchows Arch B Cell Pathol Incl Mol Pathol 62 (1) (1992) 199-206.

[47] H.H. Luu, Q. Kang, J.K. Park, W. Si, Q. Luo, W. Jiang, H. Yin, A.G. Montag, M.A. Simon, T.D. Peabody, R.C. Haydon, C.W. Rinker-Schaeffer, T.-C. He, An orthotopic model of human osteosarcoma growth and spontaneous pulmonary metastasis, Clin Exp Metastasis 22 (4) (2005) 319-329.

[48] Bérengère Gobin, Séverine Battaglia, R. Lanel, J. Chesneau, Jérôme Amiaud, F. Rédini, B. Ory, D. Heymann, NVP-BEZ235, a dual PI3K/mTOR inhibitor, inhibits osteosarcoma cell proliferation and tumor development in vivo with an improved survival rate, Cancer Lett 344 (2) (2014) 291-298.

[49] A.I. Ségaliny, A. Mohamadi, B. Dizier, A. Lokajczyk, Régis Brion, R. Lanel, Jérôme Amiaud, Céline Charrier, C. Boisson-Vidal, D. Heymann, Interleukin-34 promotes tumor progression and metastatic process in osteosarcoma through induction of angiogenesis and macrophage recruitment, Int J Cancer 137 (1) (2015) 73-85.

[50] L. Tattersall, Z. Davison, A. Gartland, in: Encyclopedia of Bone Biology, Elsevier, 2020, pp. 362-378, https://doi.org/10.1016/B978-0-12-801238-3.62259-6.

[51] D. Green, H. Eyre, A. Singh, J.T. Taylor, J. Chu, L. Jeys, V. Sumathi, A. Coonar, D. Rassl, M. Babur, D. Forster, S. Alzabin, F. Ponthan, A. McMahon, B. Bigger, T. Reekie, M. Kassiou, K. Williams, T. Dalmay, W.D. Fraser, K.G. Finegan, Targeting the MAPK7/MMP9 axis for metastasis in primary bone cancer, Oncogene 39 (33) (2020) 5553-5569.
[52] R. Lara, E. Adinolfi, C.A. Harwood, M. Philpott, J.A. Barden, F. Di Virgilio, S. McNulty, P2X7 in Cancer: From Molecular Mechanisms to Therapeutics, Front Pharmacol 11 (2020) 793.

[53] N.-i.-H. Syed, C. Kennedy, Pharmacology of P2X receptors, Wiley Interdisciplinary Reviews: Membrane Transport and Signaling 1(1) (2012) 16-30.

[54] Y.A. Fouad, C. Aanei, Revisiting the hallmarks of cancer, American Journal of Cancer Research, E-Century Publishing Corporation, 2017, pp. 1016-1036.

[55] J. Xia, X. Yu, L. Tang, G. Li, T. He, P2X7 receptor stimulates breast cancer cell invasion and migration via the AKT pathway, Oncol Rep 34 (1) (2015) 103110.

[56] B.J. Gu, J.S. Wiley, Rapid ATP-induced release of matrix metalloproteinase 9 is mediated by the P2X7 receptor, Blood 107 (12) (2006) 4946-4953.

[57] A. Gartland, K.A. Buckley, R.A. Hipskind, M.J. Perry, J.H. Tobias, G. Buell, I. Chessell, W.B. Bowler, J.A. Gallagher, Multinucleated osteoclast formation in vivo and in vitro by P2X7 receptor-deficient mice, Crit Rev Eukaryot Gene Expr 13 (2-4) (2003) 243-253.

[58] H.Z. Ke, H. Qi, A.F. Weidema, Q. Zhang, N. Panupinthu, D.T. Crawford, W.A. Grasser, V.M. Paralkar, M. Li, L.P. Audoly, C.A. Gabel, W.S.S. Jee, S.J. Dixon, S.M. Sims, D.D. Thompson, Deletion of the P2X7 nucleotide receptor reveals its regulatory roles in bone formation and resorption, Mol Endocrinol 17 (7) (2003) 1356-1367.

[59] S. Syberg, S. Petersen, J.E. Beck Jensen, A. Gartland, J. Teilmann, I. Chessell, T.H. Steinberg, P. Schwarz, N.R. Jorgensen, Genetic Background Strongly Influences the Bone Phenotype of P2X7 Receptor Knockout Mice, J Osteoporos (2012, 2012,) 391097.

[60] N. Wang, A. Agrawal, N.R. Jorgensen, A. Gartland, P2X7 receptor regulates osteoclast function and bone loss in a mouse model of osteoporosis, Sci Rep 8 (1) (2018) 3507.

[61] J. Rousseau, V. Escriou, F. Lamoureux, Régis Brion, J. Chesneau, Séverine Battaglia, Jérome Amiaud, D. Scherman, D. Heymann, F. Rédini, V. Trichet, Formulated siRNAs targeting Rankl prevent osteolysis and enhance chemotherapeutic response in osteosarcoma models, J Bone Miner Res 26 (10) (2011) 2452-2462.

[62] Z. Kun-Peng, Z. Chun-Lin, M. Xiao-Long, Z. Lei, Fibronectin-1 modulated by the long noncoding RNA OIP5-AS1/miR-200b-3p axis contributes to doxorubicin resistance of osteosarcoma cells, J Cell Physiol 234 (5) (2019) 6927-6939.

[63] X. Xu, B. Wang, Y. Xu, Expression of lysyl oxidase in human osteosarcoma and its clinical significance: a tumor suppressive role of LOX in human osteosarcoma cells, Int J Oncol 43 (5) (2013) 1578-1586.

[64] T.R. Cox, R.M.H. Rumney, E.M. Schoof, L. Perryman, A.M. Høye, A. Agrawal, D. Bird, N.A. Latif, H. Forrest, H.R. Evans, I.D. Huggins, G. Lang, R. Linding, A. Gartland, J.T. Erler, The hypoxic cancer secretome induces pre-metastatic bone lesions through lysyl oxidase, Nature 522 (7554) (2015) 106-110.

[65] D. Zhang, G. Cui, C. Sun, L. Lei, L. Lei, R.A. Williamson, Y. Wang, J. Zhang, P. Chen, A. Wang, R. Fan, S. Han, Y. Wang, J. Hu, Hypoxia promotes osteosarcoma cell proliferation and migration through enhancing platelet-derived growth factor-BB/platelet-derived growth factor receptor-beta axis, Biochem Biophys Res Commun 512 (2) (2019) 360-366.

[66] C.C. Chao, W.F. Lee, W.H. Yang, C.Y. Lin, C.K. Han, Y.L. Huang, Y.C. Fong, M.H. Wu, I.T. Lee, Y.H. Tsai, C.H. Tang, J.F. Liu, IGFBP-3 stimulates human osteosarcoma cell migration by upregulating VCAM-1 expression, Life Sci 265 (2021) 118758.

[67] K. Nakajima, T. Kidani, H. Miura, Molecular profiling of bone remodeling occurring in musculoskeletal tumors, Journal of Orthopaedic Research (2020). 